\title{
Bioconvection under uniform shear: linear stability analysis
}

\author{
YONGYUN HWANG $†$ AND T. J. PEDLEY \\ Department of Applied Mathematics and Theoretical Physics, University of Cambridge, \\ Centre for Mathematical Sciences, Wilberforce Road, Cambridge CB3 0WA, UK
}

(Received 8 November 2013 and in revised form ??)

The role of uniform shear in bioconvective instability in a shallow suspension of swimming gyrotactic cells is studied using linear stability analysis. The shear is introduced by applying a plane Couette flow, and it significantly disturbs gravitaxis of the cell. The unstably stratified basic state of the cell concentration is gradually relieved as the shear rate is increased, and it even becomes stably stratified at very large shear rates. Stability of the basic state is significantly changed. The instability at high wavenumbers is drastically damped out with the shear rate, while that at low wavenumbers is destabilised. However, at very large shear rates, the latter is also suppressed. The most unstable mode is found as a pair of streamwise uniform rolls aligned with the shear, analogous to Rayleigh-Bénard convection in plane Couette flow. To understand these findings, the physical mechanism of the bioconvective instability is reexamined with several sets of numerical experiments. It is shown that the bioconvective instability in a shallow suspension originates from three different physical processes: gravitational overturning, gyrotaxis of the cell, and negative cross diffusion flux. The first mechanism is found to rule the behavior of low-wavenumber instability whereas the last two mechanisms are mainly associated with high-wavenumber instability. With the increase of the shear rate, the former is enhanced, thereby leading to destabilisation at low wavenumbers, whereas the latter two mechanisms are significantly suppressed. For streamwise varying perturbations, shear with sufficiently large rates is also found to play a stabilising role as in Rayleigh-Bénard convection. However, at small shear rates, it destabilises these perturbations through the mechanism of overstability discussed by Hill et al. (1989). Finally, the present findings are compared with a recent experiment by Croze et al. (2010) and they are in qualitative agreement.

\section{Introduction}

Bioconvection is a pattern forming motion observed in shallow suspensions of cells which swim upward (against gravity). The up-swimming cells accumulate at the top and form a layer with dense population. If the cell concentration is great enough, the heavy layer at the top results in gravitational overturning, leading to a convection pattern analogous to that in Rayleigh-Bénard convection. Childress et al. (1975) developed a selfconsistent mathematical model in which they took the vertical drift by the up-swimming of cells into account, and showed the appearance of the gravitational instability.

The mechanism by which the individual cells swim upward is often governed by biased swimming of the given cell species in response to external stimuli such as gravity, light, and chemicals (i.e. taxes) (see also reviews by Pedley \& Kessler 1992; Hill \& Pedley

$\dagger$ Present address: Department of Civil and Environmental Engineering, Imperial College London, SW7 2AZ, UK. Email address for correspondence: y.hwang@imperial.ac.uk 
2005; Pedley 2010a). Of particular interest to the present study are algal cells such as C. Nivalis and Dunaliella, and colonies such as Volvox, that respond to gravity in the absence of flow. These cells are structurally featured to be bottom-heavy: their center of mass is located behind the center of buoyancy. Therefore, when a cell of this type is not oriented vertically, the bottom heaviness results in a gravitational torque which changes the cell's swimming direction to align with the vertical. In a moving fluid, a viscous torque originating from the shear is also applied to the cell. Therefore, in this case, the cell experiences both gravitational and viscous torques, and the swimming direction is determined by the balance between them. This process, known as 'gyrotaxis', was proposed and demonstrated by Kessler $(1984,1985,1986)$ in a series of pioneering experiments. In particular, he showed that, in the presence of a downward shear flow, the cells swim toward the region of most rapid downflow as a result of gyrotaxis.

The gyrotactic nature of the cell has been found to cause instability even in a uniform suspension, which does not exhibit the instability mechanism of gravitational overturning. Imagine a uniform suspension in which natural fluctuations create a 'blob' of cells denser than its surroundings. The blob will sink relative to its surroundings, and will create a downward shear flow in its wake. Owing to the gyrotaxis, other cells in the surroundings swim toward the blob and its wake, where the downflow is most rapid. The blob therefore becomes denser and creates more rapid downflow, resulting in instability of the suspension. The fluid motion resulting from this instability mechanism appears in the form of a bottom-standing plume, which is typically observed in relatively deep suspensions $(d>1 \mathrm{~cm}$ where $d$ is the depth of the suspension) (see e.g. Kessler 1986; Pedley \& Kessler 1992). Pedley et al. (1988) analysed this instability by describing the swimming of the cell in a deterministic manner with a prescribed translational diffusivity to take randomness in the cell motion into account. Pedley \& Kessler (1990) extended this analysis with an improved description on the cell's random behaviour observed to resemble a random walk. They introduced a quasi-steady Fokker-Planck equation for the probability density function (pdf) of the cells' orientation, which allowed them to calculate the mean cell orientation and the related translational diffusivity in a statistical manner. In that study, the effect of the cells' swimming on the fluid motions was also assessed although it was found to be negligible for the gyrotactic instability. Recently, Pedley (2010b) further extended this model by allowing the pdf of cell orientation to vary over time and space as well as its swimming direction. The model was designed to be more general than the early one, so that it can be applied to other cells such as bacteria and spermatozoa. Thus, it exhibits not only the gyrotactic instability but also the instability observed in dense bacterial suspensions and shown to be a consequence of the intrinsic stresslet of swimming cells (Simha \& Ramaswamy 2002; Dombrowski et al. 2005; Saintillan \& Shelley 2007, 2008).

The early models by Pedley et al. (1988) and Pedley \& Kessler (1990) were also used to study bioconvection in a shallow layer respectively by Hill et al. (1989) and Bees \& Hill (1998). It was shown that these analyses reasonably well describe the early stage of bioconvection: for example, the predicted critical cell concentration and the spatial wavelength show reasonable agreement with those observed in the experiment by Bees \& Hill (1997). In a shallow suspension, the up-swimming of the cell leads to gravitational instability as mentioned previously. Therefore, in this case, the gyrotactic and the gravitational overturning instability mechanisms co-exist. It has been thought that the two independent mechanisms cooperate with each other (Hill et al. 1989). However, the detailed cooperation dynamics is as yet not very well understood.

While the early studies focused mostly on suspensions in stationary fluid, many swimming microorganisms in aqueous environments are exposed to shear flow; for gyrotactic 
cells, in particular, the shear plays a critical role in their swimming behaviour. As mentioned, the swimming direction of a gyrotactic cell is determined by the balance between gravitational and viscous torques. When the shear rate is not large, the balance simply makes the cell's swimming direction tilt towards that of the shear. With an increase of the shear rate, the swimming direction is tilted more and more and the effect of the viscous torque gradually becomes dominant. When the shear rate is large enough, the viscous torque dominates over the gravitational one. In this case, the swimming direction of the cell becomes unsteady and changes periodically in time (Pedley \& Kessler 1987, 1992), similarly to that of a passive particle in a shear flow (Jeffery 1922). Owing to this behaviour, gyrotactic cells in a strong shear flow tumble and exhibit greatly reduced up-swimming velocity on average. As a consequence, dispersion of the cells in the vertical direction is significantly disturbed, leading to the formation of layers of cells in regions of strong shear (Durham et al. 2009; Ishikawa 2012).

In spite of the interesting behaviour of gyrotactic cells under shear flows, there have been few studies of how shear affects the bioconvection pattern. Only recently, this issue has been addressed in an experimental study by Croze et al. (2010), in which they examined the effect of a cross flow on a bioconvection pattern. They showed that the cross flow tilts the convection pattern in the direction of the shear. Also, with an increase of the flow rate, they observed an increase in the wavelength of the convection pattern. In particular, when the cell concentration is relatively low, the convection pattern was shown to be nearly extinguished. In spite of this interesting observation, no theoretical study which examines the role of shear in bioconvection is currently available, and thus no sound explanation of the observation has yet been provided.

The purpose of the present study is therefore to understand how shear affects the instability of a shallow suspension of gyrotactic cells within a theoretical and computational framework. To gain fundamental understanding of the role of shear, we consider a very simple flow configuration in which a uniform horizontal shear is imposed in a channel by moving the upper and lower walls in opposite directions: i.e. a plane Couette flow (see also figure 1). The mathematical description of the suspension follows Pedley (2010b) and a few approximations are then made. Particular emphasis in the analysis is given to understanding the physical mechanisms by which the shear affects bioconvective instability. From this perspective, we carefully reexamine the physical mechanisms of bioconvection, and this enables us to find an additional physical mechanism for the instability that originates from negative cross diffusion in the unstably stratified circumstances. We will see that shear orchestrates these three instability mechanisms in a different manner, resulting in intricate competition dynamics between them.

The paper is organised as follows: In $\S 2$, we introduce the equations of motion and formulate them for a linear stability analysis. Some approximations made in the present study are also discussed in this section. The parameters for the analysis are then presented in $\S 2.6$. The formulation for the linear stability analysis requires solutions of the FokkerPlanck equation, and they are then shown in $\S 3$. In $\S 4$, the results of the linear stability analysis are presented. A discussion on how shear influences bioconvective instability is then given in $\S 5$, where the results of the present study are also compared with those in Rayleigh-Bénard convection in uniform shear and those in the experiment by Croze et al. (2010). 


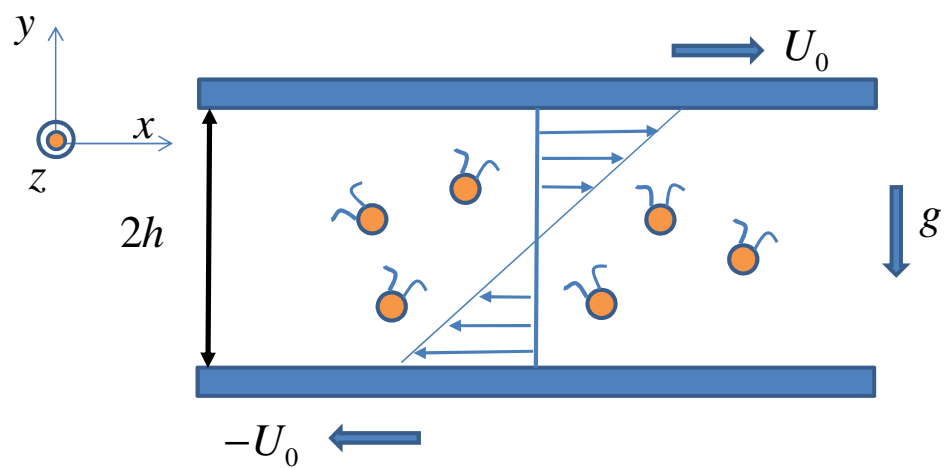

FIGURE 1. Schematic diagram of flow configuration in the present study.

\section{Problem formulation}

\subsection{Equations of motion}

We consider a fluid flow with density $\rho$ and kinematic viscosity $\nu$ in which gyrotactic cells are suspended with gravity heading downward. We denote $x^{*}, y^{*}$, and $z^{*}$ as the streamwise, wall-normal, and spanwise directions, respectively, and $t^{*}$ is the time (the superscript ${ }^{*}$ indicates dimensional variables). The suspension is bounded by two infinitely long and wide parallel walls, respectively located at $y^{*}= \pm h$, and they are set to move in opposite directions with velocity $U_{0}^{*}$. The fluid motion is described by the following equations:

$$
\begin{gathered}
\nabla^{*} \cdot \mathbf{u}^{*}=0 \\
\frac{\partial \mathbf{u}^{*}}{\partial t^{*}}+\left(\mathbf{u}^{*} \cdot \nabla^{*}\right) \mathbf{u}^{*}=-\frac{1}{\rho} \nabla^{*} p^{*}+\nu \nabla^{2} \mathbf{u}^{*}-n^{*} v g^{\prime} \mathbf{j}+\nabla^{*} \cdot \Sigma_{p}^{*},
\end{gathered}
$$

Here, $\mathbf{u}^{*}$ is the velocity, $p^{*}$ the pressure, $n^{*}$ the cell number density, $g^{\prime}=g \Delta \rho / \rho$ the reduced gravity ( $\Delta \rho$ is the density difference between cell and fluid, and $g$ the gravitational acceleration), $v$ the volume of a single cell, and $\boldsymbol{\Sigma}_{p}^{*}$ the additional stress term caused by the presence of swimming cells in the suspension. This term $\boldsymbol{\Sigma}_{p}^{*}$ was first analysed by Pedley \& Kessler (1990), in which they showed that it is dominated by the stresslets associated with the locomotion of the cells. Recent analyses have shown that this term actually plays a cruical role in generating an instability in dense suspensions of 'pusher'type swimmers (e.g. bacteria and spermatozoa), which generate thrust by pushing the fluid behind the cell body (Simha \& Ramaswamy 2002; Saintillan \& Shelley 2007, 2008; Pedley 2010b). However, in the present study, we will consider only 'puller'-type swimmers (e.g. Chlamydomonas), which swim by pulling fluid from the front to the back. In this case, the cell-stress term is not in general responsible for generating such an instability (Saintillan \& Shelley 2007, 2008; Pedley 2010b). Also, the instability in bioconvection is typically observed in dilute suspensions (typically less than $1 \%$ of cell concentration), in which this term was shown to be negligible (Pedley \& Kessler 1990). Therefore, this term will be neglected throughout the present study.

For the spatial distribution and orientation of the cells in the suspension, we consider a Smoluchowski equation which describes a conservation law for the probability density distribution as a function of spatial position $\mathbf{x}^{*}=\left(x^{*}, y^{*}, z^{*}\right)$ and of the unit vector in the swimming direction, $\mathbf{e}=\left(e_{1}, e_{2}, e_{3}\right)$ :

$$
\frac{\partial \Psi^{*}}{\partial t^{*}}+\nabla^{*} \cdot\left(\frac{d \mathbf{x}^{*}}{d t^{*}} \Psi^{*}\right)+\nabla_{e} \cdot\left(\frac{d \mathbf{e}}{d t^{*}} \Psi^{*}\right)=0,
$$


where

$$
\begin{gathered}
\frac{d \mathbf{x}^{*}}{d t^{*}}=\mathbf{u}^{*}+V_{c}^{*} \mathbf{e}-V_{s}^{*} \mathbf{j}-\mathbf{D}_{T}^{*} \cdot \nabla^{*}\left(\ln \Psi^{*}\right), \\
\frac{d \mathbf{e}}{d t^{*}}=\frac{1}{2 B}[\mathbf{j}-(\mathbf{j} \cdot \mathbf{e}) \mathbf{e}]+\frac{1}{2} \mathbf{\Omega}^{*} \wedge \mathbf{e}+\alpha_{0} \mathbf{e} \cdot \mathbf{E}^{*} \cdot\left(\mathbf{I}^{*}-\mathbf{e e}\right)-D_{R}^{*} \nabla_{e}\left(\ln \Psi^{*}\right) .
\end{gathered}
$$

Here, $\Psi^{*}\left(\mathbf{x}^{*}, \mathbf{e}, t^{*}\right)$ is the probability density distribution function, $\mathbf{i}, \mathbf{j}$, and $\mathbf{k}$ are respectively unit vectors in the streamwise, wall-normal and spanwise directions, $V_{c}^{*}$ is the cell swimming speed, $V_{s}^{*}$ the cell sedimentation speed, $\mathbf{D}_{T}^{*}$ the translational diffusivity tensor, $B=\nu \alpha_{\perp} / 2 g l$ is the gyrotactic time scale ( $l$ is the center of gravity offset), $\boldsymbol{\Omega}^{*}$ the vorticity, $\mathbf{E}^{*}$ the strain rate tensor, and $D_{R}^{*}$ the rotational diffusivity. Here, $\alpha_{0}$ in $(2.3 c)$ and $\alpha_{\perp}$ in the definition of $B$ are constants given by geometry of the cell: for example, $\alpha_{0}=0.31$ and $\alpha_{\perp}=6.8$ for $C$. Nivalis (see e.g. Pedley et al. 1988). In the present study, we assume for simplicity that the cell is completely spherical, giving $\alpha_{0}=0$ and $\alpha_{\perp}=6$. It is worth pointing out that this assumption excludes the appearance of instability mechanisms due to the cell shape: for example, rod-like swimming cells in suspension may yield the instability proposed by e.g. Koch \& Shaqfeh (1989) and Saintillan et al. (2006). However, we should also point out that, in many practical situations, this is not a great limitation, as typical gyrotactic cells are often close to a spherical shape (especially Volvox). We also note that the sedimentation speed of the cell $V_{s}^{*}$ is explicitly included, as it appears to be crucial at high shear rates.

The probability density function for the cells is decomposed such that $\Psi^{*}(\mathbf{x}, \mathbf{e}, t) \equiv$ $n^{*}\left(\mathbf{x}^{*}, t^{*}\right) f\left(\mathbf{x}^{*}, \mathbf{e}, t^{*}\right)$ where $f\left(\mathbf{x}^{*}, \mathbf{e}, t^{*}\right)$ is the probability density function only for the swimming direction of the cell, satisfying

$$
\int_{S_{e}} f\left(\mathbf{x}^{*}, \mathbf{e}, t^{*}\right) d^{2} \mathbf{e}=1,
$$

where $S_{e}$ is surface of a unit sphere on which the e-space is defined. The probability density distribution function $f\left(\mathbf{x}^{*}, \mathbf{e}, t^{*}\right)$ allows us to calculate a local ensemble average of an arbitrary variable at a given location $\mathbf{x}^{*}$ : for example, the local ensemble average of the swimming direction at a given location $\mathrm{x}^{*}$ and time $t^{*}$ is given by

$$
\langle\mathbf{e}\rangle\left(\mathbf{x}^{*}, t^{*}\right) \equiv \int_{S_{e}} \mathbf{e} f\left(\mathbf{x}^{*}, \mathbf{e}, t^{*}\right) d^{2} \mathbf{e} .
$$

It is convenient to split (2.3) into two equations respectively for $n^{*}$ and $f$. Integrating (2.3) over e-space yields the equation for $n^{*}$ :

$$
\frac{\partial n^{*}}{\partial t^{*}}+\nabla \cdot\left[n^{*}\left(\mathbf{u}^{*}+V_{c}^{*}\langle\mathbf{e}\rangle-V_{s}^{*} \mathbf{j}\right)\right]=\nabla^{*} \cdot\left(\mathbf{D}_{T}^{*} \cdot \nabla^{*} n^{*}\right)
$$

where the diffusivity tensor is approximated by the simplified expression given by Pedley \& Kessler (1990):

$$
\mathbf{D}_{T}^{*}=V_{c}^{* 2} \tau(\langle\mathbf{e e}\rangle-\langle\mathbf{e}\rangle\langle\mathbf{e}\rangle)
$$

Here, $\tau$ is the correlation time of a cell's random walk, which we will set as a constant. However, in principle, it does not need to be independent of shear rate or of the swimming direction e. We now multiply (2.5) by $f$ and subtract it from (2.3). Dividing by $n^{*}$ then yields the equation for $f$ :

$$
\frac{\partial f}{\partial t^{*}}+\left(\mathbf{u}^{*} \cdot \nabla\right) f+\nabla_{e} \cdot\left\{\frac{1}{2 B}[\mathbf{j}-(\mathbf{j} \cdot \mathbf{e}) \mathbf{e}] f+\frac{1}{2} \mathbf{\Omega}^{*} \wedge \mathbf{e} f\right\}=D_{R}^{*} \nabla_{e}^{2} f .
$$

Here, in obtaining (2.7), the translational transport by swimming and diffusion of cells is neglected as its contribution appears to be very small for the system of interest: i.e. 
$h \sim O(1 \mathrm{~cm})$ and $U_{0}^{*} / h \sim O\left(B^{-1}\right)$. For details on this approximation, see the Appendix A. We note that, under this approximation, the first two terms in (2.7) represent convective transport of $f$ by the background flow.

Regarding the equations of motion here, some additional remarks should be made. First, the randomness in the behaviour of the cell is assumed to be modeled only by the translational diffusivity tensor $\mathbf{D}_{T}^{*}$ and the rotational diffusivity $D_{R}^{*}$. However, the randomness of the real cells exists in various properties (e.g. swimming speed, size, shape, etc). In this respect, this setting itself is essentially ad hoc, as also pointed out by Pedley $(2010 a)$. Furthermore, we also assume that the rotational diffusivity $D_{R}^{*}$ does not depend on the shear rate. However, this appears not to be true: a recent experimental study has shown that the rotational diffusivity exhibits a very large value at the shear rate at which a deterministic swimmer would start to tumble (Furlan et al. 2013). Finally, it should be pointed out that the translational diffusion model (2.6) with a 'constant' $\tau$ may not be a good approximation particularly when the shear rate is quite large. This issue has been addressed by several recent studies (Hill \& Bees 2002; Malena \& Frankel 2003; Bearon et al. 2012; Croze et al. 2013), which have proposed that the spatial dispersion of the cells in strong shear flows is better described by the so-called generalised Taylor dispersion theory. We note that, in practice, the difference between the present analysis and Taylor dispersion theory appears in calculating $\mathbf{D}_{T}^{*}$. In particular, the expression (2.6) in the present study requires an experimental measurement of the correlation time scale $\tau$ as in Hill \& Häder (1997) and Vladimirov et al. (2000, 2004), and such a measurement is not available in the presence of shear. However, it has been found that (2.6) with constant $\tau$ is not a bad approximation when the shear rate is not very large (Croze et al. 2013) (see also $\S 3.1$ for further discussion).

\subsection{Non-dimensionalisation}

The governing equations (2.1), (2.2), (2.5), and (2.7) are non-dimensionalised using the following dimensionless variables:

$$
t=\frac{t^{*} D_{V}}{h^{2}}, \mathbf{x}=\frac{\mathbf{x}^{*}}{h}, \mathbf{u}=\frac{\mathbf{u}^{*} h}{D_{V}} p=\frac{p^{*} h^{2}}{\rho \mu D_{V}}, n=\frac{n^{*}}{N}, V_{c}=\frac{V_{c}^{*} h}{D_{V}}, V_{s}=\frac{V_{s}^{*} h}{D_{V}},
$$

where $D_{V}=V_{c}^{* 2} \tau$ is the scale for the translational diffusivity, and $N=1 / V \int_{\Omega} n^{*} d V$ where $\Omega$ is the domain of interest with its volume $V$. The equations of motion in terms of these variables are then given as follows:

$$
\begin{gathered}
\nabla \cdot \mathbf{u}=0 . \\
\operatorname{Sc}^{-1} \frac{\partial \mathbf{u}}{\partial t}+\operatorname{Sc}^{-1}(\mathbf{u} \cdot \nabla) \mathbf{u}=-\nabla p+\nabla^{2} \mathbf{u}-\operatorname{Ra} n \mathbf{j}, \\
\frac{\partial n}{\partial t}+\nabla \cdot\left[n\left(\mathbf{u}+V_{c}\langle\mathbf{e}\rangle-V_{s} \mathbf{j}\right)\right]=\nabla \cdot\left(\mathbf{D}_{T} \cdot \nabla n\right), \\
D_{R}^{-1} \frac{\partial f}{\partial t}+D_{R}^{-1}(\mathbf{u} \cdot \nabla) f+\nabla_{e} \cdot\left[\lambda[\mathbf{j}-(\mathbf{j} \cdot \mathbf{e}) \mathbf{e}] f+\frac{1}{2 D_{R}} \boldsymbol{\Omega} \wedge \mathbf{e} f\right]=\nabla_{e}^{2} f,
\end{gathered}
$$

with boundary conditions

$$
\begin{gathered}
\left.\mathbf{u}\right|_{y= \pm 1}=\left( \pm U_{0}, 0,0\right) \\
{\left.\left[n\left(\mathbf{u}+V_{c}\langle\mathbf{e}\rangle-V_{s} \mathbf{j}\right)-\mathbf{D}_{T} \cdot \nabla n\right]\right|_{y= \pm 1} \cdot \mathbf{j}=0,}
\end{gathered}
$$


where

$$
\mathrm{Sc} \equiv \frac{\nu}{D_{V}}, \mathrm{Ra} \equiv \frac{N v g^{\prime} h^{3}}{D_{V} \nu}, \mathbf{D}_{T}=\frac{\mathbf{D}_{T}^{*}}{D_{V}}, \lambda=\frac{1}{2 B D_{R}^{*}}, D_{R} \equiv \frac{D_{R}^{*} h^{2}}{D_{V}} .
$$

Here, Sc is the Schmidt number, Ra, the Rayleigh number, $\mathbf{D}_{T}$, the dimensionless translational diffusivity tensor, $\lambda$, the dimensionless inverse of the gyrotactic time scale, and $D_{R}$ is the dimensionless rotational diffusivity. We note that the boundary condition for $n$ imposes zero flux across the walls, which enables the total number of cells to be preserved in time. On the other hand, a boundary condition for $f$ is purposely excluded because the further approximation of $(2.9 d)$ does not allow us to prescribe it (for details, see $\S 2.3$ and $\S 2.4)$. For this reason, we simply assume that $(2.9 d)$ holds even at the walls although this is not technically correct. From this viewpoint, it should be pointed out that prescribing the boundary condition for $f$ might allow one to describe the reported interaction of swimming cells with the solid boundary (e.g. Kantsler et al. 2013). However, this issue is beyond the scope of the present study.

\subsection{Basic state}

We calculate a basic state, about which we will add small perturbations. Geometrical homogeneity of the given flow configuration in the streamwise and spanwise directions gives

$$
\frac{\partial}{\partial x}=\frac{\partial}{\partial z}=0, \mathbf{u}_{\mathbf{0}}=(\bar{U}, 0,0) .
$$

We first rescale the base-flow velocity $\bar{U}$ with the upper-wall velocity, so that the Reynolds number for the given shear is extracted: i.e. $\mathbf{u}_{\mathbf{0}}=(\operatorname{ScRe} U, 0,0)$ where $\operatorname{Re}=U_{0}^{*} h / \nu$ is the Reynolds number. The equations for the basic state are then given as

$$
\begin{gathered}
\frac{d^{2} U}{d y^{2}}=0, \\
\frac{d P_{0}}{d y}=-\operatorname{Ra} n_{0}, \\
{\left[V_{c}\left\langle e_{2}\right\rangle_{0}-V_{s}\right] \frac{d n_{0}}{d y}=D_{T 0}^{22} \frac{d^{2} n_{0}}{d y^{2}},} \\
\nabla_{e} \cdot\left[\lambda[\mathbf{j}-(\mathbf{j} \cdot \mathbf{e}) \mathbf{e}] f_{0}+\frac{S}{2} \boldsymbol{\Omega}_{0} \wedge \mathbf{e} f_{0}\right]=\nabla_{e}^{2} f_{0},
\end{gathered}
$$

with boundary conditions,

$$
\begin{gathered}
\left.U\right|_{y= \pm 1}= \pm 1 \\
\left.\left(V_{c}\left\langle e_{2}\right\rangle_{0}-V_{s}\right) n_{0}\right|_{y= \pm 1}-\left.D_{T 0}^{22} \frac{d n_{0}}{d y}\right|_{y= \pm 1}=0 .
\end{gathered}
$$

Here, $S\left(\equiv \mathrm{ScRe} / D_{R}=U_{0}^{*} / D_{R}^{*} h\right)$ is the dimensionless shear rate normalised by the rotational diffusivity, $P_{0}$ is the basic-state pressure, and the subscript 0 in $\left\langle e_{2}\right\rangle_{0}$ and $D_{T 0}^{22}$ indicates statistical properties obtained with $f_{0}$. We note that $(2.11 d)$ does not contain any partial derivatives in $\mathbf{x}$ and that it depends only on $\mathbf{e}$. This allows us to solve it separately from the other equations as shown in $\S 3.1$. Once $f_{0}(\mathbf{e})$ is obtained, it can be used to build the following solutions of $(2.11 a)$ and $(2.11 c)$ :

$$
\begin{gathered}
U(y)=y, \\
n_{0}(y)=N_{0} e^{\kappa y},
\end{gathered}
$$


with

$$
N_{0}=\frac{\kappa}{\sinh \kappa}, \kappa=\frac{V_{c}\left\langle e_{2}\right\rangle_{0}-V_{s}}{D_{T}^{22}} .
$$

where $N_{0}$ is a normalisation constant such that $1 / V \int_{\Omega} n_{0} d V=1$.

\subsection{Linearised equations for small perturbations}

Now, we consider a small perturbation around the basic state:

$$
\begin{aligned}
& \mathbf{u}=\mathbf{u}_{0}(\mathbf{x})+\epsilon \mathbf{u}^{\prime}(\mathbf{x}, t)+O\left(\epsilon^{2}\right), p=P_{0}(\mathbf{x})+\epsilon p^{\prime}(\mathbf{x}, t)+O\left(\epsilon^{2}\right), \\
& n=n_{0}(\mathbf{x})+\epsilon n^{\prime}(\mathbf{x}, t)+O\left(\epsilon^{2}\right), f=f_{0}(\mathbf{e})+\epsilon f^{\prime}(\mathbf{x}, \mathbf{e}, t)+O\left(\epsilon^{2}\right),
\end{aligned}
$$

where $\mathbf{u}^{\prime}=\left(u^{\prime}, v^{\prime}, w^{\prime}\right)$. The linearised equations of motions are then given as

$$
\begin{aligned}
& \frac{\partial u^{\prime}}{\partial x}+\frac{\partial v^{\prime}}{\partial y}+\frac{\partial w^{\prime}}{\partial z}=0 \\
& \mathrm{Sc}^{-1} \frac{\partial u^{\prime}}{\partial t}+\operatorname{Re} U \frac{\partial u^{\prime}}{\partial x}+\operatorname{Re} v^{\prime} \frac{\partial U}{\partial y}=-\frac{\partial p^{\prime}}{\partial x}+\nabla^{2} u^{\prime} \\
& \mathrm{Sc}^{-1} \frac{\partial v^{\prime}}{\partial t}+\operatorname{Re} U \frac{\partial v^{\prime}}{\partial x}=-\frac{\partial p^{\prime}}{\partial y}+\nabla^{2} v^{\prime}-\operatorname{Ra} n^{\prime} \\
& \mathrm{Sc}^{-1} \frac{\partial w^{\prime}}{\partial t}+\operatorname{Re} U \frac{\partial w^{\prime}}{\partial x}=-\frac{\partial p^{\prime}}{\partial z}+\nabla^{2} w^{\prime} \\
& \frac{\partial n^{\prime}}{\partial t}+\operatorname{ScRe} U \frac{\partial n^{\prime}}{\partial x}+V_{c}\left\langle e_{1}\right\rangle_{0} \frac{\partial n^{\prime}}{\partial x}+\left(V_{c}\left\langle e_{2}\right\rangle_{0}-V_{s}\right) \frac{\partial n^{\prime}}{\partial y}+V_{c}\left\langle e_{3}\right\rangle_{0} \frac{\partial n^{\prime}}{\partial z} \\
& +\left(v^{\prime}+V_{c}\left\langle e_{2}\right\rangle^{\prime}\right) \frac{d n_{0}}{d y}+V_{c} n_{0}\left(\frac{\partial\left\langle e_{1}\right\rangle^{\prime}}{\partial x}+\frac{\partial\left\langle e_{2}\right\rangle^{\prime}}{\partial y}+\frac{\partial\left\langle e_{3}\right\rangle^{\prime}}{\partial z}\right) \\
& -\frac{\partial D_{T}^{12^{\prime}}}{\partial x} \frac{\partial n_{0}}{\partial y}-\frac{\partial D_{T}^{22^{\prime}}}{\partial y} \frac{\partial n_{0}}{\partial y}-\frac{\partial D_{T}^{32^{\prime}}}{\partial z} \frac{\partial n_{0}}{\partial y}-D_{T}^{22^{\prime}} \frac{d^{2} n_{0}}{d y^{2}} \\
& -D_{T 0}^{11} \frac{\partial^{2} n^{\prime}}{\partial x^{2}}-2 D_{T 0}^{12} \frac{\partial^{2} n^{\prime}}{\partial x \partial y}-D_{T 0}^{22} \frac{\partial^{2} n^{\prime}}{\partial y^{2}}-D_{T 0}^{33} \frac{\partial^{2} n^{\prime}}{\partial z^{2}}=0 . \\
& D_{R}^{-1} \frac{\partial f^{\prime}}{\partial t}+S U \frac{\partial f^{\prime}}{\partial x}+\nabla_{e} \cdot\left[\lambda[\mathbf{j}-(\mathbf{j} \cdot \mathbf{e}) \mathbf{e}] f^{\prime}+\frac{S}{2} \boldsymbol{\Omega}_{0} \wedge \mathbf{e} f^{\prime}\right]-\nabla_{e}^{2} f^{\prime} \\
& =-D_{R}^{-1} \nabla_{e} \cdot\left[\frac{1}{2} \mathbf{\Omega}^{\prime} \wedge \mathbf{e} f_{0}\right]
\end{aligned}
$$

with boundary conditions

$$
\begin{gathered}
\left.u^{\prime}\right|_{y= \pm 1}=\left.v^{\prime}\right|_{y= \pm 1}=\left.w^{\prime}\right|_{y= \pm 1}=0 \\
{\left[\left(V_{c}\left\langle e_{2}\right\rangle_{0}-V_{s}\right) n^{\prime}+V_{c}\left\langle e_{2}\right\rangle^{\prime} n_{0}\right]-D_{T 0}^{22} \frac{\partial n^{\prime}}{\partial y}-\left.D_{T}^{22^{\prime}} \frac{d n_{0}}{d y}\right|_{y= \pm 1}=0 .}
\end{gathered}
$$

Here, the superscript ' for $\left\langle e_{i}\right\rangle$ and $D_{T}^{i j}(i, j=1,2,3)$ indicates the statistical properties obtained with $f^{\prime}$.

It appears that performing a linear stability analysis directly with (2.14) is quite difficult as $f^{\prime}$ is six-dimensional owing to its dependence on $t, \mathbf{x}$, and $\mathbf{e}$. A numerical approach evidently requires extremely expensive computational cost. Given the number of parameters in the present system, such an approach would not be feasible in practice. Therefore, some approximations should be made to overcome this difficulty. In the present study, 
we assume that $f^{\prime}$ is quasi-steady and quasi-unform, yielding the following equation for $f^{\prime}$ instead of $(2.14 f)$ :

$$
\nabla_{e} \cdot\left[\lambda[\mathbf{j}-(\mathbf{j} \cdot \mathbf{e}) \mathbf{e}] f^{\prime}+\frac{S}{2} \boldsymbol{\Omega}_{0} \wedge \mathbf{e} f^{\prime}\right]-\nabla_{e}^{2} f^{\prime}=-\frac{1}{2 D_{R}} \nabla_{e} \cdot\left[\boldsymbol{\Omega}^{\prime} \wedge \mathbf{e} f_{0}\right] .
$$

It should be mentioned that this approximation would be strictly valid only if $f^{\prime}$ is slowly varying in time and space: $f^{\prime}(t, \mathbf{x}, \mathbf{e})=f^{\prime}(T, \mathbf{X}, \mathbf{e})$ where $T=\delta t$ and $\mathbf{X}=\delta \mathbf{x}$ with $\delta \ll 1$. Therefore, the approximation may not be good when the vortical perturbation $\boldsymbol{\Omega}^{\prime}$ carries rapidly varying spatio-temporal fluctuation of high wavenumber or frequency. However, such a vortical perturbation would probably be damped by viscosity, thus the approximation may not significantly disturb the range where the instability appears. We also note that the approximation removes the partial derivatives in $t$ and $\mathbf{x}$ in $(2.14 f)$. Thus, the initial and boundary conditions for these independent variables cannot be set. Also, this approximation makes the present approach practically identical to that in Pedley \& Kessler (1990).

Under this approximation, the left-hand side of (2.15) turns out to be linear and depends only on the swimming direction vector e. On the other hand, the right-hand side is simply a linear combination of e-dependent functions with coefficients $\omega_{1}^{\prime}, \omega_{2}^{\prime}$, and $\omega_{3}^{\prime}$ which are respectively the streamwise, wall-normal, and spanwise components of $\boldsymbol{\Omega}^{\prime}\left(=\left(\omega_{1}^{\prime}, \omega_{2}^{\prime}, \omega_{3}^{\prime}\right)\right)$. This suggests that the solution of $(2.15)$ is written in the following form:

$$
f^{\prime}(\mathbf{x}, \mathbf{e}, t)=\frac{1}{D_{R}}\left[\omega_{1}^{\prime}(\mathbf{x}, t) f_{\omega_{1}}^{\prime}(\mathbf{e})+\omega_{2}^{\prime}(\mathbf{x}, t) f_{\omega_{2}}^{\prime}(\mathbf{e})+\omega_{3}^{\prime}(\mathbf{x}, t) f^{\prime}{ }_{\omega_{3}}(\mathbf{e})\right] .
$$

Here, $f^{\prime}{ }_{\omega_{i}}(\mathbf{e})(i=1,2,3)$ is the solution of (2.15) depending only on e when $D_{R}=1$, $\omega_{i}^{\prime}=1$, and $\omega_{j}^{\prime}=0$ for $j \neq i$. The solution form (2.16) implies that $\langle\mathbf{e}\rangle^{\prime}$ and $\mathbf{D}_{T}^{\prime}$ in $(2.14 e)$ are also written as a linear combination of $\omega_{i}^{\prime}$. Examining numerical solution of $f^{\prime}{ }_{\omega_{i}}(\mathbf{e})$ (see $§ 3.2$ ) allows us to write $\langle\mathbf{e}\rangle^{\prime}$ and $\mathbf{D}_{T}^{\prime}$ as follows:

$$
\begin{aligned}
& \left\langle\mathbf{e}_{1}\right\rangle^{\prime}=\frac{\zeta_{1}}{D_{R}} \omega_{3}^{\prime},\left\langle\mathbf{e}_{2}\right\rangle^{\prime}=\frac{\zeta_{2}}{D_{R}} \omega_{3}^{\prime},\left\langle\mathbf{e}_{3}\right\rangle^{\prime}=\frac{\zeta_{3}}{D_{R}} \omega_{1}^{\prime}+\frac{\zeta_{4}}{D_{R}} \omega_{2}^{\prime}, \\
& D_{T}^{12^{\prime}}=\frac{\zeta_{5}}{D_{R}} \omega_{3}^{\prime}, D_{T}^{22^{\prime}}=\frac{\zeta_{6}}{D_{R}} \omega_{3}^{\prime}, D_{T}^{32^{\prime}}=\frac{\zeta_{7}}{D_{R}} \omega_{1}^{\prime}+\frac{\zeta_{8}}{D_{R}} \omega_{2}^{\prime},
\end{aligned}
$$

where $\zeta_{i}$ are essentially obtained from the first- and the second-order moments of $f^{\prime}{ }_{\omega_{i}}(\mathbf{e})$, and they are given in (3.2).

Now, we substitute (2.17) into (2.14e) and eliminate $p^{\prime}$ in (2.14) using the standard procedure (see e.g. Schmid \& Henningson 2001). Then, the following wall-normal velocity $\left(v^{\prime}\right)$ and vorticity $\left(\eta^{\prime}\right)$ form of the linearised equations is obtained:

$$
\begin{aligned}
& {\left[\left(\mathrm{Sc}^{-1} \frac{\partial}{\partial t}+\operatorname{Re} U \frac{\partial}{\partial x}\right) \nabla^{2}-\operatorname{Re} \frac{d^{2} U}{d y^{2}} \frac{\partial}{\partial x}-\nabla^{4}\right] v^{\prime}+\operatorname{Ra}\left(\frac{\partial^{2} n^{\prime}}{\partial x^{2}}+\frac{\partial^{2} n^{\prime}}{\partial z^{2}}\right)=0} \\
& \left(\mathrm{Sc}^{-1} \frac{\partial}{\partial t}+\operatorname{Re} U \frac{\partial}{\partial x}-\nabla^{2}\right) \eta^{\prime}+\operatorname{Re} \frac{d U}{d y} \frac{\partial v^{\prime}}{\partial z}=0 \\
& \frac{\partial n^{\prime}}{\partial t}+\operatorname{ScRe} U \frac{\partial n^{\prime}}{\partial x}+V_{c}\left\langle e_{1}\right\rangle_{0} \frac{\partial n^{\prime}}{\partial x}+\left(V_{c}\left\langle e_{2}\right\rangle_{0}-V_{s}\right) \frac{\partial n^{\prime}}{\partial y}+V_{c}\left\langle e_{3}\right\rangle_{0} \frac{\partial n^{\prime}}{\partial z} \\
& +v^{\prime} \frac{d n_{0}}{d y}+G_{1} \zeta_{2} \omega_{3}^{\prime} \frac{d n_{0}}{d y}+G_{1}\left[n_{0}\left(\zeta_{1} \frac{\partial \omega_{3}^{\prime}}{\partial x}+\zeta_{2} \frac{\partial \omega_{3}^{\prime}}{\partial y}+\zeta_{3} \frac{\partial \omega_{1}^{\prime}}{\partial z}+\zeta_{4} \frac{\partial \omega_{2}^{\prime}}{\partial z}\right)\right] \\
& -G_{2}\left[\zeta_{5} \frac{\partial \omega_{3}^{\prime}}{\partial x} \frac{d n_{0}}{d y}+\zeta_{6} \frac{\partial \omega_{3}^{\prime}}{\partial y} \frac{d n_{0}}{d y}+\left(\zeta_{7} \frac{\partial \omega_{1}^{\prime}}{\partial z}+\zeta_{8} \frac{\partial \omega_{2}^{\prime}}{\partial z}\right) \frac{d n_{0}}{d y}+\zeta_{6} \omega_{3}^{\prime} \frac{d^{2} n_{0}}{d y^{2}}\right]
\end{aligned}
$$




$$
-D_{T 0}^{11} \frac{\partial^{2} n^{\prime}}{\partial x^{2}}-2 D_{T 0}^{12} \frac{\partial^{2} n^{\prime}}{\partial x \partial y}-D_{T 0}^{22} \frac{\partial^{2} n^{\prime}}{\partial y^{2}}-D_{T 0}^{33} \frac{\partial^{2} n^{\prime}}{\partial z^{2}}=0,
$$

where

$$
G_{1} \equiv \frac{V_{c}}{D_{R}}=\frac{V_{c}^{*}}{D_{R}^{*} h}, G_{2} \equiv \frac{1}{D_{R}}=\frac{D_{V}}{D_{R}^{*} h^{2}} .
$$

Here, $G_{1}$ describes the importance of swimming relative to rotational diffusion, and $G_{2}$ represents the importance of translational diffusion relative to rotational diffusion. We note that, for negligibly small sedimentation (i.e. $\left.V_{s}^{*} / V_{c}^{*} \ll 1\right), G_{1} \sim O\left(\kappa G_{2}\right)$. Also, for a given $\lambda=1 /\left(2 B D_{R}^{*}\right), G_{2} \sim O\left(B V_{c}^{* 2} \tau / h^{2}\right)$, retrieving the dimensionless gyrotaxis parameter given in Hill et al. (1989) and Bees \& Hill (1998). As we shall see in $§ 5.1, G_{1}$ and $G_{2}$ are dimensionless indicators of the contribution that the gyrotactic nature of the cell makes to instability.

The linear system (2.18) does not depend on the swimming direction vector e. The geometrical homogeneity in the streamwise and spanwise directions allows us to consider the following normal-mode solution of (2.18),

$$
\begin{aligned}
& v^{\prime}(x, y, z, t)=\hat{v}(y) e^{i(\alpha x+\beta z-\omega t)}+c . c, \eta^{\prime}(x, y, z, t)=\hat{\eta}(y) e^{i(\alpha x+\beta z-\omega t)}+c . c \\
& n^{\prime}(x, y, z, t)=\hat{n}(y) e^{i(\alpha x+\beta z-\omega t)}+c . c,
\end{aligned}
$$

where $\alpha$ and $\beta$ are respectively the streamwise and spanwise wavenumbers, and $\omega$ is the frequency. We note that $\hat{u}, \hat{v}$, and $\hat{w}$ are obtained from

$$
\left(\begin{array}{c}
\hat{u} \\
\hat{v} \\
\hat{w}
\end{array}\right)=\frac{1}{k^{2}}\left(\begin{array}{cc}
i \alpha \mathcal{D} & -i \beta \\
k^{2} & 0 \\
i \beta \mathcal{D} & i \alpha
\end{array}\right)\left(\begin{array}{l}
\hat{v} \\
\hat{\eta}
\end{array}\right)
$$

where $\mathcal{D}=d / d y$ and $k=\sqrt{\alpha^{2}+\beta^{2}}$. Using $(2.20)$, the vorticity components $\hat{\omega}_{1}, \hat{\omega}_{2}$ and $\hat{\omega}_{3}$ in $(2.18)$ are given as

$$
\left(\begin{array}{c}
\hat{\omega}_{1} \\
\hat{\omega}_{2} \\
\hat{\omega}_{3}
\end{array}\right)=\left(\begin{array}{c}
\mathcal{D} \hat{w}-i \beta \hat{v} \\
\hat{\eta} \\
i \alpha \hat{v}-\mathcal{D} \hat{u}
\end{array}\right)=\frac{1}{k^{2}}\left(\begin{array}{cc}
-i \beta\left(k^{2}-\mathcal{D}^{2}\right) & i \alpha \mathcal{D} \\
0 & k^{2} \\
i \alpha\left(k^{2}-\mathcal{D}^{2}\right) & i \beta \mathcal{D}
\end{array}\right)\left(\begin{array}{l}
\hat{v} \\
\hat{\eta}
\end{array}\right) .
$$

The equations for linear stability are then obtained as follows:

$$
i \omega\left(\begin{array}{ccc}
\mathrm{Sc}^{-1}\left(k^{2}-\mathcal{D}^{2}\right) & 0 & 0 \\
0 & \mathrm{Sc}^{-1} & 0 \\
0 & 0 & I
\end{array}\right)\left(\begin{array}{c}
\hat{v} \\
\hat{\eta} \\
\hat{n}
\end{array}\right)=\left(\begin{array}{ccc}
L_{O S} & 0 & k^{2} \mathrm{Ra} \\
i \beta \operatorname{Re} \mathcal{D} U & L_{S Q} & 0 \\
\mathcal{D} n_{0}+L_{C}^{v} & L_{C}^{\eta} & L_{C}
\end{array}\right)\left(\begin{array}{c}
\hat{v} \\
\hat{\eta} \\
\hat{n}
\end{array}\right),
$$

where

$$
\begin{gathered}
L_{O S}=i \alpha \operatorname{Re} U\left(k^{2}-\mathcal{D}^{2}\right)+i \alpha R e \mathcal{D}^{2} U+\left(k^{2}-\mathcal{D}^{2}\right)^{2} \\
L_{S Q}=i \alpha \operatorname{Re} U+\left(k^{2}-\mathcal{D}^{2}\right) \\
L_{C}^{v}=\left[G_{1}\left(\zeta_{2} \mathcal{D} n_{0} \frac{i \alpha}{k^{2}}-n_{0}\left(\zeta_{1} \frac{\alpha^{2}}{k^{2}}-\zeta_{2} \mathcal{D} \frac{i \alpha}{k^{2}}-\zeta_{3} \frac{\beta^{2}}{k^{2}}\right)\right)-G_{2}\left(\zeta_{6} \mathcal{D}^{2} n_{0} \frac{i \alpha}{k^{2}}\right.\right. \\
\left.\left.-\zeta_{5} \mathcal{D} n_{0} \frac{\alpha^{2}}{k^{2}}+\zeta_{6} \mathcal{D} n_{0} \frac{i \alpha}{k^{2}} \mathcal{D}+\zeta_{7} \mathcal{D} n_{0} \frac{\beta^{2}}{k^{2}}\right)\right]\left(k^{2}-\mathcal{D}^{2}\right), \\
L_{C}^{\eta}=\left[G_{1}\left(\zeta_{2} \mathcal{D} n_{0} \frac{i \beta}{k^{2}}-n_{0}\left(\zeta_{1} \frac{\alpha \beta}{k^{2}}-\zeta_{2} \mathcal{D} \frac{i \beta}{k^{2}}+\zeta_{3} \frac{\alpha \beta}{k^{2}}\right)\right)-G_{2}\left(\zeta_{6} \mathcal{D}^{2} n_{0} \frac{i \beta}{k^{2}}+\zeta_{5} \mathcal{D} n_{0} \frac{\alpha \beta}{k^{2}}\right.\right. \\
\left.\left.-\zeta_{6} \frac{i \beta}{k^{2}} \mathcal{D} n_{0} \mathcal{D}+\zeta_{7} \mathcal{D} n_{0} \frac{\alpha \beta}{k^{2}}\right)\right] \mathcal{D}-G_{2} \zeta_{8} \mathcal{D} n_{0} i \beta
\end{gathered}
$$




$$
\begin{aligned}
L_{C} & =i \alpha \operatorname{ScRe} U+i \alpha V_{c}\left\langle e_{1}\right\rangle+\left(V_{c}\left\langle e_{2}\right\rangle-V_{s}\right) D+i \beta V_{c}\left\langle e_{3}\right\rangle \\
& +\alpha^{2} D_{T 0}^{11}-2 i \alpha D_{T 0}^{12} \mathcal{D}-D_{T 0}^{22} D^{2}+\beta^{2} D_{T 0}^{33} .
\end{aligned}
$$

with boundary conditions,

$$
\begin{gathered}
\left.\hat{v}\right|_{y= \pm 1}=\left.\mathcal{D} \hat{v}\right|_{y= \pm 1}=0,\left.\hat{\eta}\right|_{y= \pm 1}=0 \\
{\left[\left(V_{c}\left\langle e_{2}\right\rangle_{0}-V_{s}-i \alpha D_{T 0}^{12}\right) \hat{n}-D_{T 0}^{22} \mathcal{D} \hat{n}+\left(G_{1} \zeta_{2} n_{0}-G_{2} \zeta_{6} \mathcal{D} n_{0}\right)\right.} \\
\left.\left(\frac{i \alpha}{k^{2}}\left(k^{2}-\mathcal{D}^{2}\right) \hat{v}+\frac{i \beta}{k^{2}} \mathcal{D} \hat{\eta}\right)\right]_{y= \pm 1}=0 .
\end{gathered}
$$

Here, $L_{O S}$ is the Orr-Sommerfeld operator, $L_{S Q}$ the Squire operator, $L_{C}$ the advectiondiffusion operator for the cell number density, $L_{C}^{v}$ the coupling operator between $\hat{v}$ and $\hat{n}$, and $L_{C}^{\eta}$ the coupling operator between $\hat{\eta}$ and $\hat{n}$. We note that if $G_{1}=G_{2}=0$, the form of the linearised equation is identical to that for Rayleigh-Bénard convection with a cross flow (e.g. Gallagher \& Mercer 1965; Kelly 1992; Jerome et al. 2012). Computation for the linear instability by excluding the operators for $\eta$ (i.e. $L_{S Q}, L_{C}^{\eta}$ and $i \beta \operatorname{Re} \mathcal{D} U$ ) show that their contribution is small, as one might have expected from Squire's theorem (Drazin \& Reid 1981; Schmid \& Henningson 2001) and the fact that the wall-normal vorticity $\eta^{\prime}$ in the Squire equation $(2.18 b)$ becomes only a passive variable if $L_{C}^{\eta}=0$. However, it should be mentioned that these operators induce large temporal transient growth of the perturbation even for the stable linear system and that they play a crucial role in the disturbance amplification in bypass transition (Ellingsen \& Palm 1975; Butler \& Farrell 1992; Reddy \& Henningson 1993) and fully-developed turbulent flows (Hwang \& Cossu 2010). This instability, which grows algebraically in inviscid flows (Ellingsen \& Palm 1975), can be typically analysed by the so-called 'non-modal' stability analysis (Schmid \& Henningson 2001; Schmid 2007). However, this mechanism is active only for $R e \geqslant 20$, at least (Schmid \& Henningson 2001), whereas the Reynolds number in the present study is less than 20 (see table 2). Therefore, this transiently growing instability can be safely neglected.

\subsection{Numerical methods}

The stability of (2.22) is investigated numerically. First, the solutions of the unsteady Fokker-Planck equations $(2.11 d)$ and (2.15) are obtained as follows: The swimming vector is presented in spherical coordinates such that $\mathbf{e}=(\sin \theta \cos \phi, \cos \theta, \sin \theta \sin \phi)$ with $0 \leqslant \theta<\pi$ and $0 \leqslant \phi<2 \pi$. Discretisation in the $\theta$ direction is conducted using secondorder central differences, while the $\phi$ direction is discretised using the Fourier-Galerkin method. Time integration is performed semi-implicity: the rotational diffusion term is advanced using the second-order Crank-Nicolson method, while the remaining terms are marched with a third-order low-storage Runge-Kutta method. The unsteady numerical solutions reach a steady state within $t^{*} \simeq 10 D_{R}^{*}$. The numerical solutions for steady $f_{0}$ and $f^{\prime}$ are taken at $t^{*}=20 D_{R}^{*}$, which was found to be sufficient to ensure convergence of the solutions. The computations are carried out with $N_{\theta} \times N_{\phi}=100 \times 48\left(N_{\theta}\right.$ and $N_{\phi}$ are the number of grid points respectively in the $\theta$ and $\phi$ directions), and the results do not show any difference from those with a lower resolution $\left(N_{\theta} \times N_{\phi}=80 \times 32\right)$. The first-order and second-order statistics such as $\langle\mathbf{e}\rangle$ and $\langle\mathbf{e e}\rangle$ from $f_{0}(\mathbf{e})$ in the present study show very good agreement with those in Bees et al. (1998) where the solution is obtained with a highly truncated series expansion of spherical harmonic functions.

The equations for linear stability (2.22) are discretised using a Chebyshev-collocation method (Weideman \& Reddy 2000). The boundary conditions are implicitly imposed 


\begin{tabular}{lccc}
\hline Parameter & Description & Reference Value & Units \\
$\rho$ & Fluid density & 1 & $\mathrm{~g} / \mathrm{cm}^{3}$ \\
$g$ & Gravitational acceleration & 980 & $\mathrm{~cm}^{2}$ \\
$\nu$ & Dynamic viscosity & 0.01 & $\mathrm{~cm}^{2} / \mathrm{s}$ \\
$d(=2 h)$ & Depth of suspension & 0.5 & $\mathrm{~cm}$ \\
$N$ & Cell mean number density & $1 \times 10^{4} \sim 1 \times 10^{8}$ & $\mathrm{cells} / \mathrm{cm}^{3}$ \\
$\Delta \rho / \rho$ & Relative cell density & 0.05 & - \\
$v$ & Cell volume & $2.1 \times 10^{-9}$ & $\mathrm{~cm}^{3}$ \\
$g^{\prime}(=g \Delta \rho / \rho)$ & Relative gravity & 49 & $\mathrm{~cm} / \mathrm{s}^{2}$ \\
$l$ & Center of gravity offset & $10^{-4}$ & $\mathrm{~cm}$ \\
$\alpha_{0}$ & Cell geometry constant & 0 & - \\
$\alpha_{\perp}$ & Cell geometry constant & 6 & - \\
$B$ & gyrotactic time scale & 3.4 & $\mathrm{sec}$ \\
$U_{0}^{*} / h$ & Shear rate & $0 \sim 0.74$ & $1 / \mathrm{s}$ \\
$V_{c}^{*}$ & Swimming speed & $6.3 \times 10^{-3}$ & $\mathrm{~cm} / \mathrm{s}$ \\
$V_{s}^{*}$ & Sedimentation speed & $6 \times 10^{-4}$ & $\mathrm{~cm} / \mathrm{s}$ \\
$\tau$ & Correlation time scale & 5 & $\mathrm{~s}$ \\
$D_{V}^{*}\left(=V_{c}^{* 2} \tau\right)$ & Nominal translation cell diffusivity & $1.98 \times 10^{-4}$ & $\mathrm{~cm} / \mathrm{s}$ \\
$D_{R}^{*}$ & Rotational diffusivity & 0.067 & $1 / \mathrm{s}$
\end{tabular}

TABLE 1. Parameters and their reference values in the present study. Most of the parameters for the cell properties are taken from the data for C. Nivalis (Pedley \& Kessler 1990; Bees \& Hill 1998; Pedley 2010b).

\begin{tabular}{lcc}
\hline Parameter & Description & Reference Value \\
Sc & Schmidt number & 50 \\
$\operatorname{Ra}$ & Rayleigh number & $10^{1} \sim 10^{5}$ \\
$S$ & Shear rate normalised by $D_{R}^{*}$ & $0 \sim 11$ \\
$\operatorname{Re}$ & Reynolds number of base-flow shear & $0 \sim 18$ \\
$G_{1}$ & see $(2.18)$ & 0.38 \\
$G_{2}\left(=1 / D_{R}\right)$ & see $(2.18)$ & 0.047 \\
$\lambda$ & gyrotactic time scale normalised by $D_{R}^{*}$ & 2.2 \\
$D_{R}$ & Rotational diffusivity normalised by $D_{V}^{*}$ & 21
\end{tabular}

TABLE 2. Dimensionless parameters and their values in the present study.

on the discretised operator, so that spurious eigenvalues do not appear. The discretised eigenvalue problem is solved using the function eig in Matlab. The computation is performed with $N_{y}=101$, showing no difference from the results with $N_{y}=201$. The computed eigenvalues for $\mathrm{Ra}=0$ show excellent agreement with those of the OrrSommerfeld-Squire operators (e.g. Schmid \& Henningson 2001), and they also show very good agreement with those in the classical Rayleigh-Bénard problem (e.g. Drazin \& Reid 1981) when $\operatorname{Re}=0, \mathrm{Sc}=1, G_{1}=G_{2}=0, \mathcal{D} n_{0}(y)=1$ and $\left.n^{\prime}\right|_{y= \pm 1}=0$ are set. Finally, the neutral stability curves for $S=0$ are compared with those in Bees \& Hill (1998), also showing good agreement.

\subsection{Parameters}

A list of parameters and their reference values are summarised in table 1. As in previous studies (e.g. Pedley \& Kessler 1990; Bees \& Hill 1998; Pedley 2010b), most of the parameters are taken from the values relevant for $C$. Nivalis except $\alpha_{0}$ and $\alpha_{\perp}$, which are set for a spherical cell. We note that the correlation time scale for the translational 
(a)

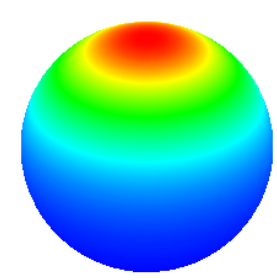

(c)

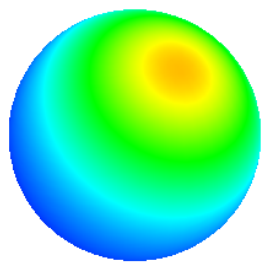

(b)

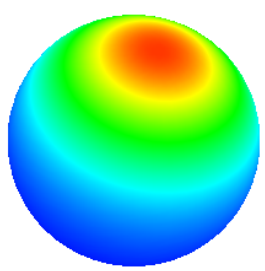

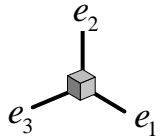

Figure 2. Basic state of probability density function of the cell orientation vector $f_{0}(\mathbf{e})$ $(\lambda=2.2)$ : (a) $S=0 ;(b) S=2 ;(c) S=4 ;(d) S=8$. All the functions exhibit planar symmetry about the $e_{1}-e_{2}$ plane.
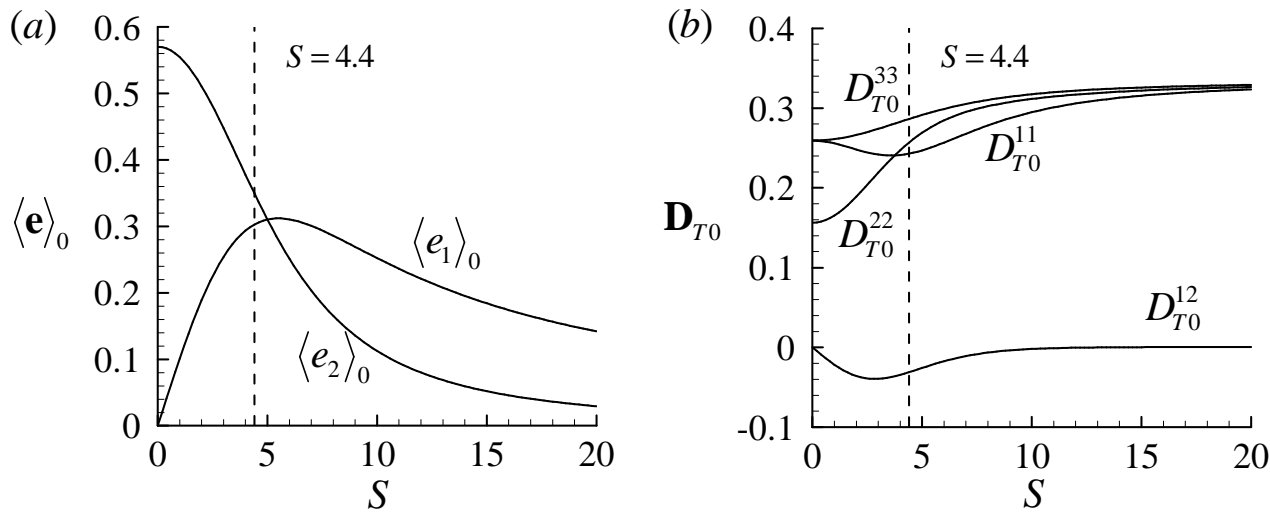

FiguRE 3. (a) Mean cell swimming vector and $(b)$ diffusivity tensor $(\lambda=2.2)$. Here, the dashed vertical lines indicate $S=4.4$ at which the deterministic swimmer begins to experience tumbling. The terms not shown here are zero in numerical precision.

diffusivity model is chosen as $\tau=5 \mathrm{~s}$ instead of $\tau=1.3 \mathrm{~s}$ used in e.g. Pedley (2010b) because this value was shown to give better agreement with the experimental data (Bees $\&$ Hill 1998). The depth of the suspension and the shear rate are chosen by considering typical conditions for laboratory experiments (Durham et al. 2009; Croze et al. 2010), so that the results can be compared with the experimental data when available. The dimensionless parameters and their reference values are given in table 2 .

\section{Fokker-Planck equation}

\subsection{Basic state}

The solution of the Fokker-Planck equation $f_{0}(\mathbf{e})$ under uniform shear was extensively discussed in Bees et al. (1998). Therefore, only a brief discussion on the computed $f_{0}(\mathbf{e})$ is given here. However, here we also present the structure of $f_{0}(\mathbf{e})$, which was not shown by 
Bees et al. (1998). Figure 2 visualises $f_{0}(\mathbf{e})$ for several values of the shear rate parameter $S$. For $S=0$, the numerical solution is identical to the analytic solution in Pedley \& Kessler $(1990)\left(f_{0}(\theta)=\lambda /(4 \pi \sinh \lambda) e^{\lambda \cos \theta}\right)$, which is symmetric about the $e_{2}$-axis (figure $2 a)$. As the shear rate $S$ increases, the peak location of $f_{0}(\mathbf{e})$ is tilted in the direction of shear. However, with increasing $S$, the peak value decreases and $f_{0}(\mathbf{e})$ tends to be distributed more uniformly.

The mean swimming direction vector $\langle\mathbf{e}\rangle_{0}$ and the diffusivity tensor $\mathbf{D}_{T 0}$ are computed using the calculated $f_{0}(\mathbf{e})$. Figure $3(a)$ shows the mean swimming direction vector. For $S=0$, only the vertical component appears, implying that the cells swim only upward in this case. With an increase of $S$, this component of the mean swimming vector gradually decreases. On the other hand, the streamwise component increases until $S \simeq 5$, after which it also decays with shear. We note that the shear rate at which the deterministic swimmer would experience tumbling is $S_{c}=4.4$, suggesting that the decay of the streamwise component is probably associated with this. For very large shear rate $(S>10)$, both components become small although the streamwise one is more persistent than the vertical one. The spanwise component is found to be zero for all $S$ as is to be expected by symmetry. The diffusivity tensor is also shown in figure $3(b)$. When the shear rate is small, $\mathbf{D}_{T 0}$ generally appears to be highly anisotropic. For $S=0, D_{T 0}^{22}$ is smaller than $D_{T 0}^{11}$ and $D_{T 0}^{33}$, and $D_{T 0}^{12}=0$. With the increase of the shear rate from $S=0, D_{T 0}^{22}$ and $D_{T 0}^{33}$ increase while $D_{T 0}^{11}$ and $D_{T 0}^{12}$ decrease. In particular, $D_{T 0}^{12}$ becomes negative in this range, and this is later shown to play an important role in instability (see $§ 5.1$ ). We note that the $D_{T 0}^{12}$ being negative implies a relation between the first- and second-order moments of the e-distribution: i.e. $\left\langle e_{1} e_{2}\right\rangle_{0}<\left\langle e_{1}\right\rangle_{0}\left\langle e_{2}\right\rangle_{0}$. Such characteristics in the statistical moments should originate in the random walk model considered in the present study. However, as the use of the Fokker-Planck equation has been shown to be a reasonable approximation for the random walk in the cell orientation (e.g. Vladimirov et al. 2004), the negative $D_{T 0}^{12}$ in this range would not be unrealistic. The behaviour of $D_{T 0}^{11}$ and $D_{T 0}^{12}$ is changed around $S=3 \sim 5$ similarly to the streamwise component of the mean swimming vector (figure $3 a$ ). However, $D_{T 0}^{22}$ and $D_{T 0}^{33}$ monotonically increase with the shear rate. For very large shear rate $(S>10)$, the diffusivity tensor becomes nearly isotropic (i.e. $D_{T 0}^{11} \simeq D_{T 0}^{22} \simeq D_{T 0}^{33}$ and $D_{T 0}^{12} \simeq 0$ ).

It should be mentioned that the diffusivity tensor shown here (eq. (2.6)) may not be a good approximation except when $S$ is quite small (Bearon et al. 2012). The present results show a large discrepancy with those from generalised Taylor dispersion theory particularly when $S \gtrsim 10$ (Croze et al. 2013, see also Appendix B for this estimation). In this regime, The present results show a large discrepancy with those from generalised Taylor dispersion theory (Croze et al. 2013): for example, generalised Taylor dispersion theory typically shows quite small $D_{T 0}^{11}$ and $D_{T 0}^{33}$ in this case (Hill \& Bees 2002; Bearon et al. 2012). Therefore, care needs to be taken in interpreting the results of the linear stability analysis for $S>10$. However, as we shall see in $\S 4$, the behaviour of the instability in this regime is found not to be very interesting: the instability is strongly damped out by the stable stratified $n_{0}(y)$ for $S>11$ (see also figure 7 ). Therefore, the discrepancy with Taylor dispersion theory does not greatly limit the present analysis.

\subsection{Perturbed state}

We have shown that the first- and second-order moments of $f^{\prime}(\mathbf{x}, \mathbf{e}, t)$ are composed of a linear combination of $f_{\omega_{i}}^{\prime}(\mathbf{e})$ with $\omega_{i}^{\prime}$ (see (2.16)). Figure 4 shows $f_{\omega_{i}}^{\prime}(\mathbf{e})$ for $i=1,2,3$ for $S=0$ and $S=4$. We note that $\int_{S_{e}} f_{\omega_{i}}^{\prime}(\mathbf{e}) d^{2} \mathbf{e}=0$ from $(2.4 a)$. Therefore, $f_{\omega_{i}}^{\prime}(\mathbf{e})$ typically shows two extrema that are respectively positive and negative. For $S=0$, non-trivial solutions are obtained for $f_{\omega_{1}}^{\prime}(\mathbf{e})$ (figure $4 a$ ) and $f_{\omega_{3}}^{\prime}(\mathbf{e})$ (figure $4 c$ ) whereas $f_{\omega_{2}}^{\prime}(\mathbf{e})=0$ 
(a)

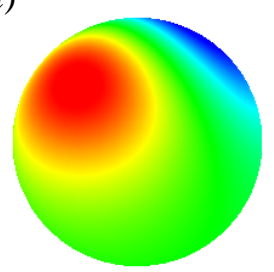

(d)

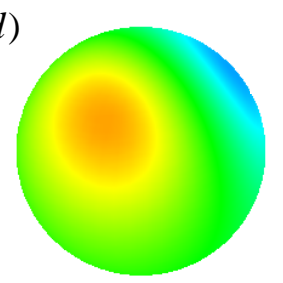

(b)

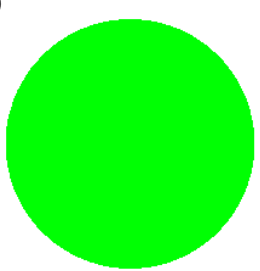

(e)

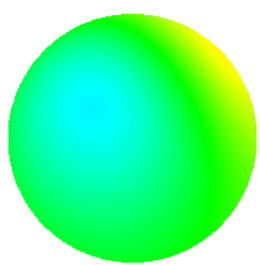

(c)

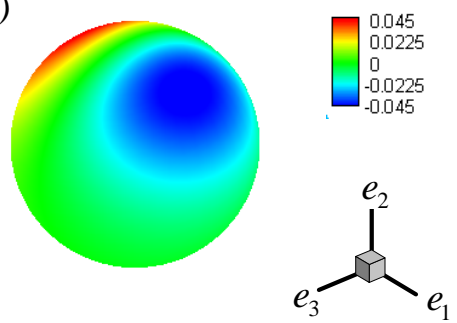

$(f)$

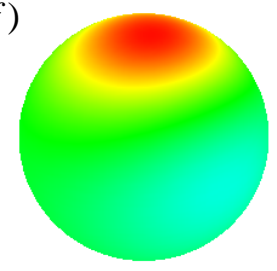

Figure 4. Perturbed probability density function of the cell orientation vector for $(a, b, c)$ $S=0$ and $(d, e, f) S=4\left(\lambda=2.2, D_{R}=1\right):(a, d) f_{\omega_{1}}^{\prime}(\mathbf{e}) ;(b, e) f_{\omega_{2}}^{\prime}(\mathbf{e}) ;(c, f) f_{\omega_{3}}^{\prime}(\mathbf{e})$.

(figure $4 b$ ). This indicates that the presence of small $\omega_{1}$ and $\omega_{3}$ would change $f$ at leading order whereas small $\omega_{2}$ would not. We also note that $f_{\omega_{1}}^{\prime}(\mathbf{e})$ is identical to $f_{\omega_{3}}^{\prime}(\mathbf{e})$ when it rotates $90^{\circ}$ clockwise about the $e_{2}$-axis. In the presence of shear, $f_{\omega_{1}}^{\prime}(\mathbf{e})$ and $f_{\omega_{3}}^{\prime}(\mathbf{e})$ are tilted towards the shear direction like $f_{0}(\mathbf{e})$ (figures $4 d$ and $f$ ). In this case, $f_{\omega_{2}}^{\prime}(\mathbf{e})$ is found to be non-trivial (figure $4 e$ ), implying that small $\omega_{2}$ would affect $f$ at the leading order.

Using $f_{\omega_{i}}^{\prime}(\mathbf{e})$, the contribution of each $\omega_{i}^{\prime}$ component to $\langle\mathbf{e}\rangle^{\prime}$ and $\langle\mathbf{e e}\rangle^{\prime}$ is computed as shown in figure 5 . For a given shear rate $S$, the presence of a small $\omega_{1}$ component $\left(\omega_{1}^{\prime}\right)$ is found to change only $\left\langle e_{3}\right\rangle$ (figure $5 a$ ) while $\left\langle e_{1}\right\rangle$ and $\left\langle e_{2}\right\rangle$ are not changed. The presence of small $\omega_{1}$ would yield a viscous torque around the $e_{1}$-axis, leading to the non-trivial $\left.\left\langle e_{3}\right\rangle\right|_{\omega_{1}} ^{\prime}$. We note that the small $\omega_{1}$ should also lead to a small change of $\left\langle e_{1}\right\rangle$ and $\left\langle e_{2}\right\rangle$. However, unlike $\left\langle e_{3}\right\rangle$, the change of $\left\langle e_{1}\right\rangle$ and $\left\langle e_{2}\right\rangle$ with respect to $\omega_{1}$ is expected to exhibit even symmetry about $\omega_{1}=0$. Therefore, their leading-order change due to the small $\omega_{1}$ $\left(\left.\left\langle e_{1}\right\rangle^{\prime}\right|_{\omega_{1}}\right.$ and $\left.\left.\left\langle e_{2}\right\rangle^{\prime}\right|_{\omega_{1}}\right)$ should be zero as also seen in the present numerical result. Such an even symmetry is also expected for $\left\langle e_{1} e_{1}\right\rangle,\left\langle e_{1} e_{2}\right\rangle,\left\langle e_{2} e_{2}\right\rangle$, and $\left\langle e_{3} e_{3}\right\rangle$, giving zeros for their leading-order change (i.e. $\left.\left\langle e_{1} e_{1}\right\rangle^{\prime}\right|_{\omega_{1}}=\left.\left\langle e_{1} e_{2}\right\rangle^{\prime}\right|_{\omega_{1}}=\left.\left\langle e_{2} e_{2}\right\rangle^{\prime}\right|_{\omega_{1}}=\left.\left\langle e_{3} e_{3}\right\rangle^{\prime}\right|_{\omega_{1}}=0$; see also figure $5 b)$. Exactly the same behaviour is seen in the leading-order change of the first- and second-order moments due to a small $\omega_{2}$. In this case, only $\left.\left\langle e_{3}\right\rangle^{\prime}\right|_{\omega_{2}}$ (figure $5 c$ ), $\left.\left\langle e_{2} e_{3}\right\rangle^{\prime}\right|_{\omega_{2}}$, and $\left.\left\langle e_{3} e_{1}\right\rangle^{\prime}\right|_{\omega_{2}}$ (figure $5 d$ ) are found to be non-zero. On the other hand, adding a small $\omega_{3}^{\prime}$ appears to be different from the cases with $\omega_{1}^{\prime}$ and $\omega_{2}^{\prime}$. For a given shear rate $S$, adding a small $\omega_{3}$ yields the leading-order change in $\left\langle e_{1}\right\rangle\left(\left.\left\langle e_{1}\right\rangle^{\prime}\right|_{\omega_{3}}\right)$ and $\left\langle e_{2}\right\rangle\left(\left.\left\langle e_{2}\right\rangle^{\prime}\right|_{\omega_{3}}\right)$ whereas the leading-order change in $\left\langle e_{3}\right\rangle\left(\left.\left\langle e_{3}\right\rangle^{\prime}\right|_{\omega_{3}}\right)$ is found to be zero for all the shear rates considered (figure $5 e$ ). We note that this behaviour is essentially due to the fact that the presence of $\omega_{3}$ does not yield viscous torques around the $e_{1}$ - and $e_{2}$-axes. For the same reason, $\left.\left\langle e_{1} e_{3}\right\rangle^{\prime}\right|_{\omega_{3}}$ and $\left.\left\langle e_{2} e_{3}\right\rangle^{\prime}\right|_{\omega_{3}}$ are found to be zero (figure $5 f$ ).

These findings suggest that $\langle\mathbf{e}\rangle^{\prime}$ and $\langle\mathbf{e e}\rangle^{\prime}$ are written as the sum of all the contributions due to $\omega_{1}^{\prime}, \omega_{2}^{\prime}$, and $\omega_{3}^{\prime}$ :

$$
\left\langle e_{1}\right\rangle^{\prime}=\frac{\left.\left\langle e_{1}\right\rangle^{\prime}\right|_{\omega_{3}}}{D_{R}} \omega_{3}^{\prime},\left\langle e_{2}\right\rangle^{\prime}=\frac{\left.\left\langle e_{2}\right\rangle^{\prime}\right|_{\omega_{3}}}{D_{R}} \omega_{3}^{\prime},\left\langle e_{3}\right\rangle^{\prime}=\frac{\left.\left\langle e_{3}\right\rangle^{\prime}\right|_{\omega_{1}}}{D_{R}} \omega_{1}^{\prime}+\frac{\left.\left\langle e_{3}\right\rangle^{\prime}\right|_{\omega_{2}}}{D_{R}} \omega_{2}^{\prime},
$$


(a)

$\left.\langle\mathbf{e}\rangle\right|_{\omega}$

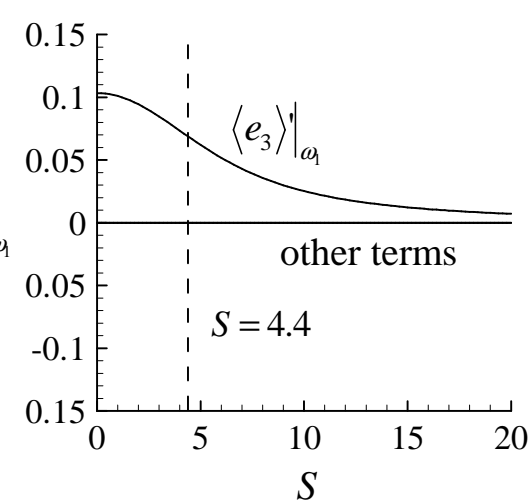

(c)

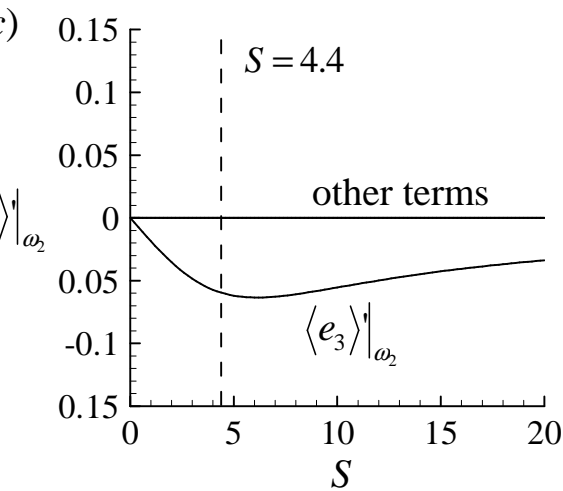

$(e)$

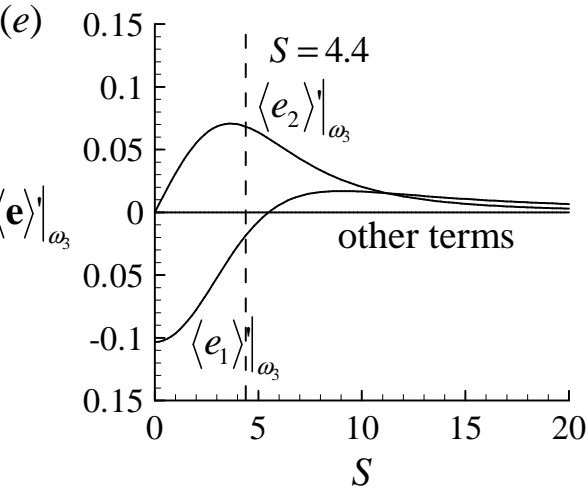

(b)

$\langle$ ee $\rangle$

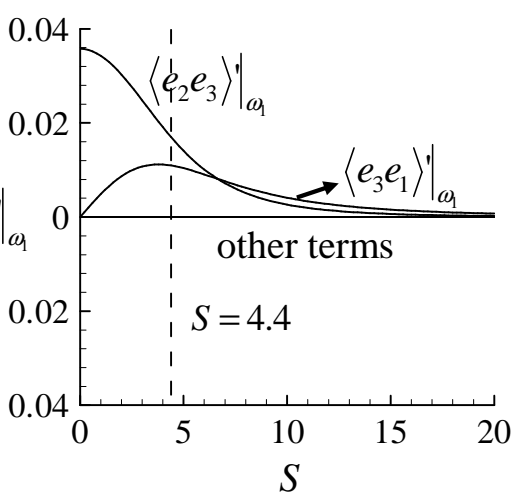

(d)

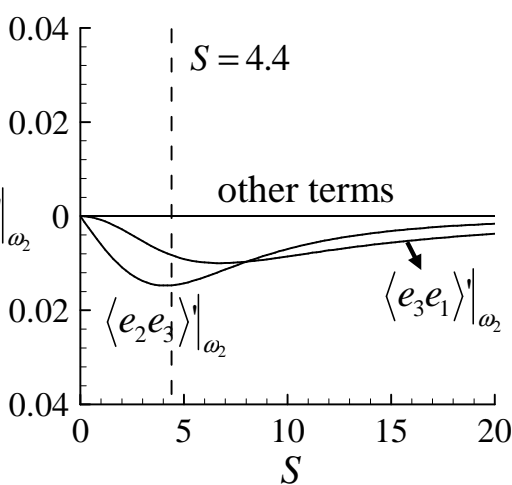

$(f)$

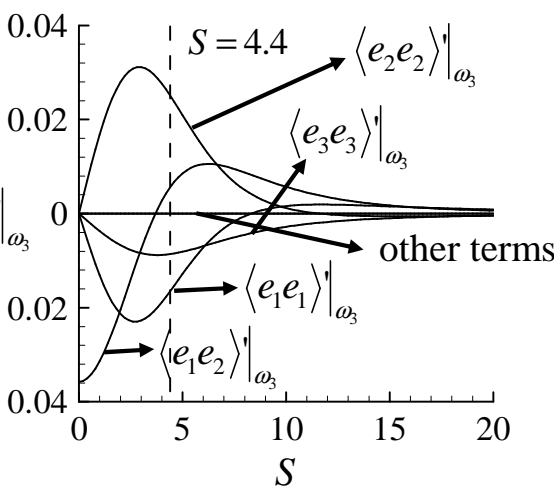

Figure 5. Componentwise contribution to $(a, c, e)$ first- and $(b, d, f)$ second-order moments of $f^{\prime}$ from $(a, b) f_{\omega_{1}}^{\prime}(\mathbf{e}),(c, d) f_{\omega_{2}}^{\prime}(\mathbf{e})$, and $(e, f) f_{\omega_{3}}^{\prime}(\mathbf{e})(\lambda=2.2)$. Here, the dashed vertical lines indicate $S=4.4$ at which the deterministic swimmer begins to experience tumbling.

$$
\left\langle e_{1} e_{2}\right\rangle^{\prime}=\frac{\left.\left\langle e_{1} e_{2}\right\rangle^{\prime}\right|_{\omega_{3}}}{D_{R}} \omega_{3}^{\prime},\left\langle e_{2} e_{2}\right\rangle^{\prime}=\frac{\left.\left\langle e_{2} e_{2}\right\rangle^{\prime}\right|_{\omega_{3}}}{D_{R}} \omega_{3}^{\prime},\left\langle e_{3} e_{2}\right\rangle^{\prime}=\frac{\left.\left\langle e_{3} e_{2}\right\rangle^{\prime}\right|_{\omega_{1}}}{D_{R}} \omega_{1}^{\prime}+\frac{\left.\left\langle e_{3} e_{2}\right\rangle^{\prime}\right|_{\omega_{2}}}{D_{R}} \omega_{2}^{\prime},
$$

Here, we note that the $\left.\langle\mathbf{e}\rangle\right|_{\omega_{i}} ^{\prime}$ and $\left.\langle\mathbf{e e}\rangle\right|_{\omega_{i}} ^{\prime}$ are equivalent to the coefficients given in Pedley \& Kessler (1990) (e.g. $J_{1}, J_{2}$, etc.): for example, the $J_{1}$ and $J_{2}$ in that study are retrieved such that $J_{1}=-\left.2 \lambda\left\langle e_{1}\right\rangle\right|_{\omega_{3}} ^{\prime}$ and $J_{2}=-\left.2 \lambda\left\langle e_{1} e_{2}\right\rangle\right|_{\omega_{3}} ^{\prime}$. The analytic solutions of the perturbed Fokker-Planck equation in Pedley \& Kessler (1990) at $S=0$ give $J_{1}=0.45$ and $J_{2}=0.16$ for $\lambda=2.2$, which are in very good agreement with $J_{1}=0.455$ and 
(a)

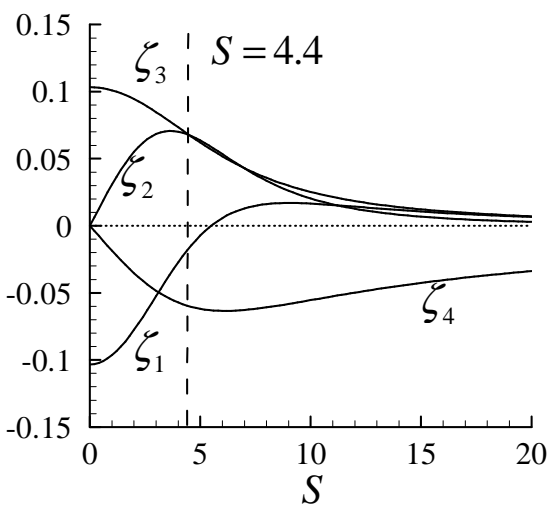

(b)

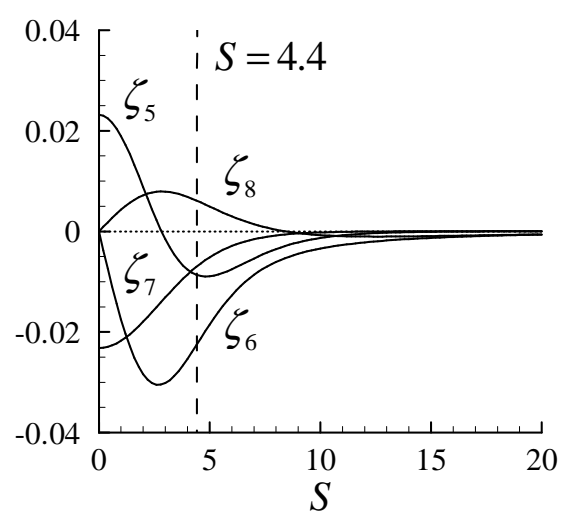

FiguRE 6 . Dependence of $\zeta_{i}$ on $S:(a) \zeta_{1}, \ldots, \zeta_{4} ;(b) \zeta_{5}, \ldots, \zeta_{8}$. Here, the dashed vertical lines indicate $S=4.4$ at which the deterministic swimmer begins to experience tumbling.

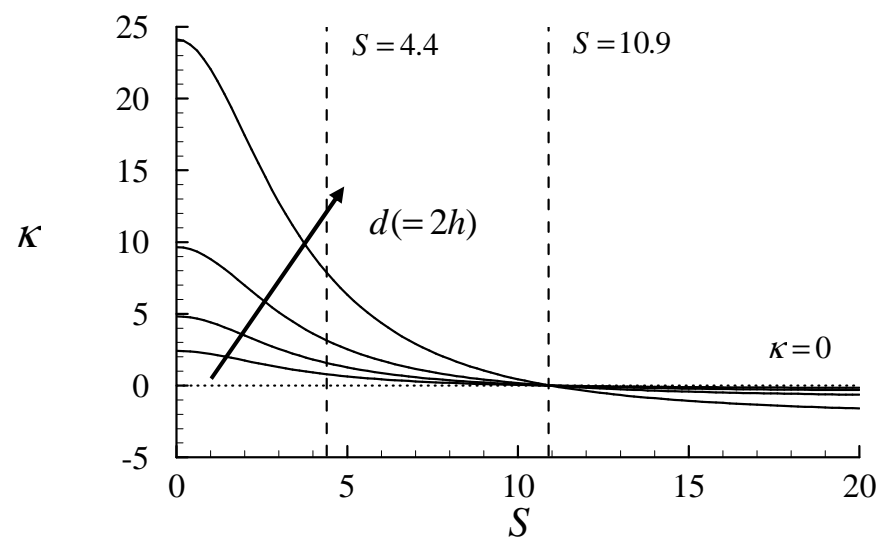

FIgURE 7. Dependence of the stratification parameter $\kappa$ on the depth of suspension $(d(=2 h)=0.05,0.1,0.2,0.5 \mathrm{~cm})$ and the shear rate $S$. Here, the vertical dashed line in the left indicates the shear rate $(S=4.4)$ at which a deterministic swimmer begins to rumble, while the one in the right is the shear rate $(S=10.9)$ where $\kappa=0$ due to sedimentation of the swimmer.

$J_{2}=0.157$. Since $\mathbf{D}_{T}^{\prime}=\langle\mathbf{e e}\rangle^{\prime}-\langle\mathbf{e}\rangle_{0}\langle\mathbf{e}\rangle^{\prime}-\langle\mathbf{e}\rangle^{\prime}\langle\mathbf{e}\rangle_{0}$, the $\zeta_{i}{ }^{\prime}$ s in (2.17) are given as follows:

$$
\begin{gathered}
\zeta_{1}=\left.\left\langle e_{1}\right\rangle^{\prime}\right|_{\omega_{3}}, \zeta_{2}=\left.\left\langle e_{2}\right\rangle^{\prime}\right|_{\omega_{3}}, \zeta_{3}=\left.\left\langle e_{3}\right\rangle^{\prime}\right|_{\omega_{1}}, \zeta_{3}=\left.\left\langle e_{3}\right\rangle^{\prime}\right|_{\omega_{2}}, \\
\zeta_{5}=\left.\left\langle e_{1} e_{2}\right\rangle^{\prime}\right|_{\omega_{3}}-\left.\left\langle e_{1}\right\rangle_{0}\left\langle e_{2}\right\rangle^{\prime}\right|_{\omega_{3}}-\left.\left\langle e_{2}\right\rangle_{0}\left\langle e_{1}\right\rangle^{\prime}\right|_{\omega_{3}}, \\
\zeta_{6}=\left.\left\langle e_{2} e_{2}\right\rangle^{\prime}\right|_{\omega_{3}}-\left.2\left\langle e_{2}\right\rangle_{0}\left\langle e_{2}\right\rangle^{\prime}\right|_{\omega_{3}}, \\
\zeta_{7}=\left.\left\langle e_{3} e_{2}\right\rangle^{\prime}\right|_{\omega_{1}}-\left.\left\langle e_{2}\right\rangle_{0}\left\langle e_{3}\right\rangle^{\prime}\right|_{\omega_{1}}, \\
\zeta_{8}=\left.\left\langle e_{3} e_{2}\right\rangle^{\prime}\right|_{\omega_{2}}-\left.\left\langle e_{2}\right\rangle_{0}\left\langle e_{3}\right\rangle^{\prime}\right|_{\omega_{2}} .
\end{gathered}
$$

The computed $\zeta_{i}$ 's are shown in figure 6 . 

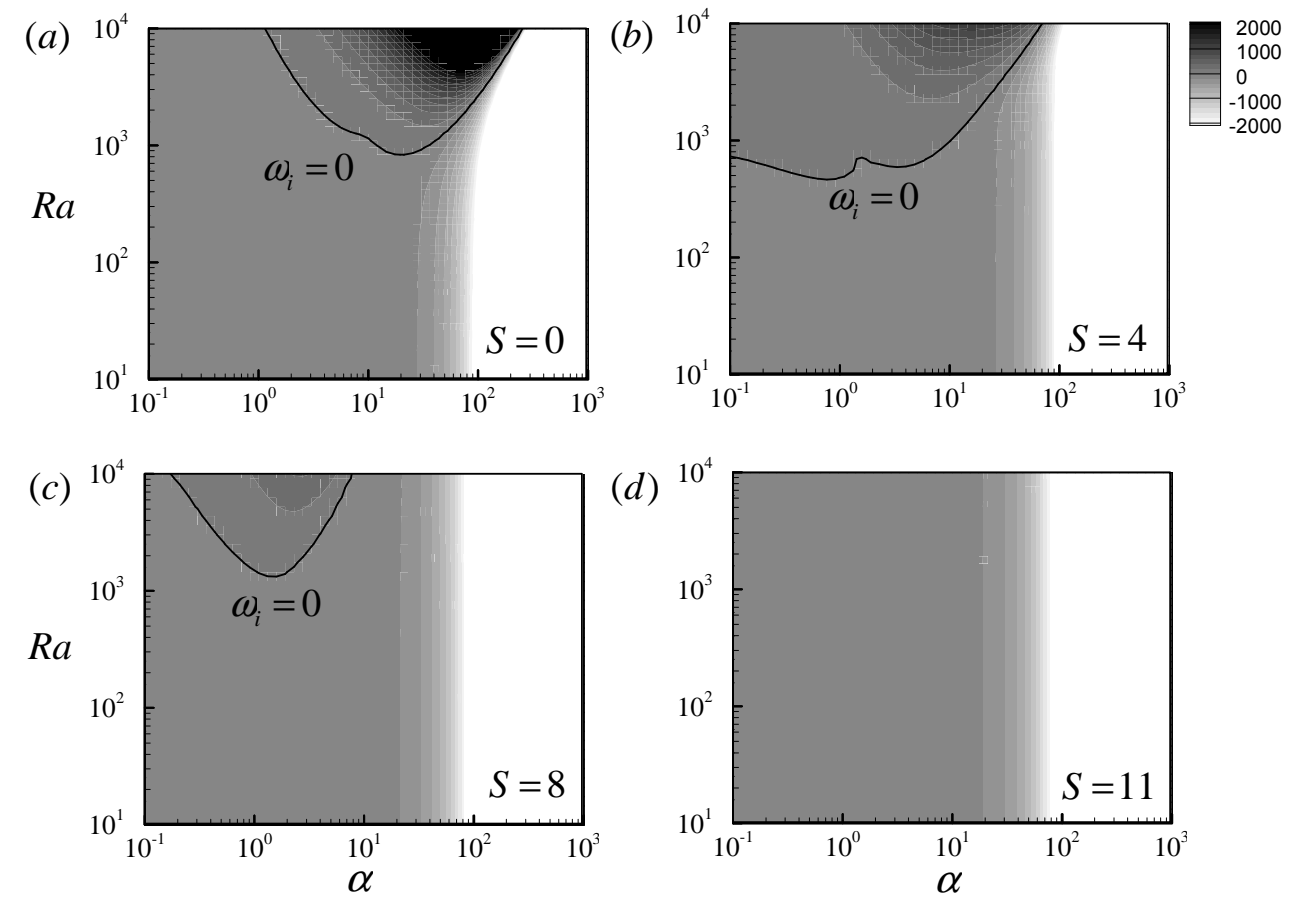

FIgURE 8. Contour of $\omega_{i}$ of the most unstable mode and its neutral stability curve in the Ra- $\alpha$ plane for $\alpha \neq 0$ and $\beta=0$ : (a) $S=0 ;(b) S=4 ;(c) S=8$; (d) $S=11$.

\section{Results}

\subsection{Basic state}

To complete the basic state in $(2.12), \kappa$ should be first calculated from the solution of the Fokker-Planck equation $f_{0}(\mathbf{e})$. The calculated $\kappa$ is reported in figure 7 . For a given depth of suspension, $\kappa$ decreases with the shear rate $S$. This indicates that the cells would accumulate less at the top and that the thickness of the dense cell layer would increase with the shear rate. The decrease of $\kappa$ is not surprising because the mean up-swimming velocity $V_{c}\left\langle e_{2}\right\rangle_{0}$ decays with the shear rate (see also figure $3 a$ ). The decrease is particularly drastic for shear rates less than $S \simeq 4.4$, the value at which a deterministic cell would begin to tumble. For $S \gtrsim 10.9, \kappa$ becomes negative because the up-swimming velocity becomes even smaller than the sedimentation velocity $\left(V_{c}\left\langle e_{2}\right\rangle_{0}<V_{s}\right)$. Therefore, in this regime, $n_{0}(y)$ becomes stably stratified. Finally, we note that $\kappa \sim O(h)$ (from $(2.12 c)$ and the non-dimensionalisation (2.8)): with the increase of the depth, $\kappa$ generally becomes large.

\subsection{Neutral stability curve}

Linear stability analysis is performed with the reference parameters in tables 1 and 2 . We remind the reader that $\alpha$ and $\beta$ are respectively the streamwise and spanwise wavenumbers. We first consider two-dimensional modes (i.e. $\alpha \neq 0$ and $\beta=0$ ). Figure 8 shows contours of the growth rate $\omega_{i}$ and the corresponding neutral stability curve $\left(\omega_{i}=0\right)$ in the Ra- $\alpha$ plane for $S=0,4,8,11$. Here, note that $S=11$ gives slightly stable stratification of $n_{0}(y)$. In the absence of shear $(S=0)$, the instability appears at $\mathrm{Ra}_{c} \simeq$ 830 with the critical wavenumber $\alpha_{c} \simeq 19$ (figure 8a), giving $N_{c}=1.05 \times 10^{6}$ cells $/ \mathrm{cm}^{3}$ and $\lambda_{c}=0.1 \mathrm{~cm}$. These values compare reasonably well with $N_{c}=1.0 \times 10^{6} \mathrm{cells} / \mathrm{cm}^{3}$ 

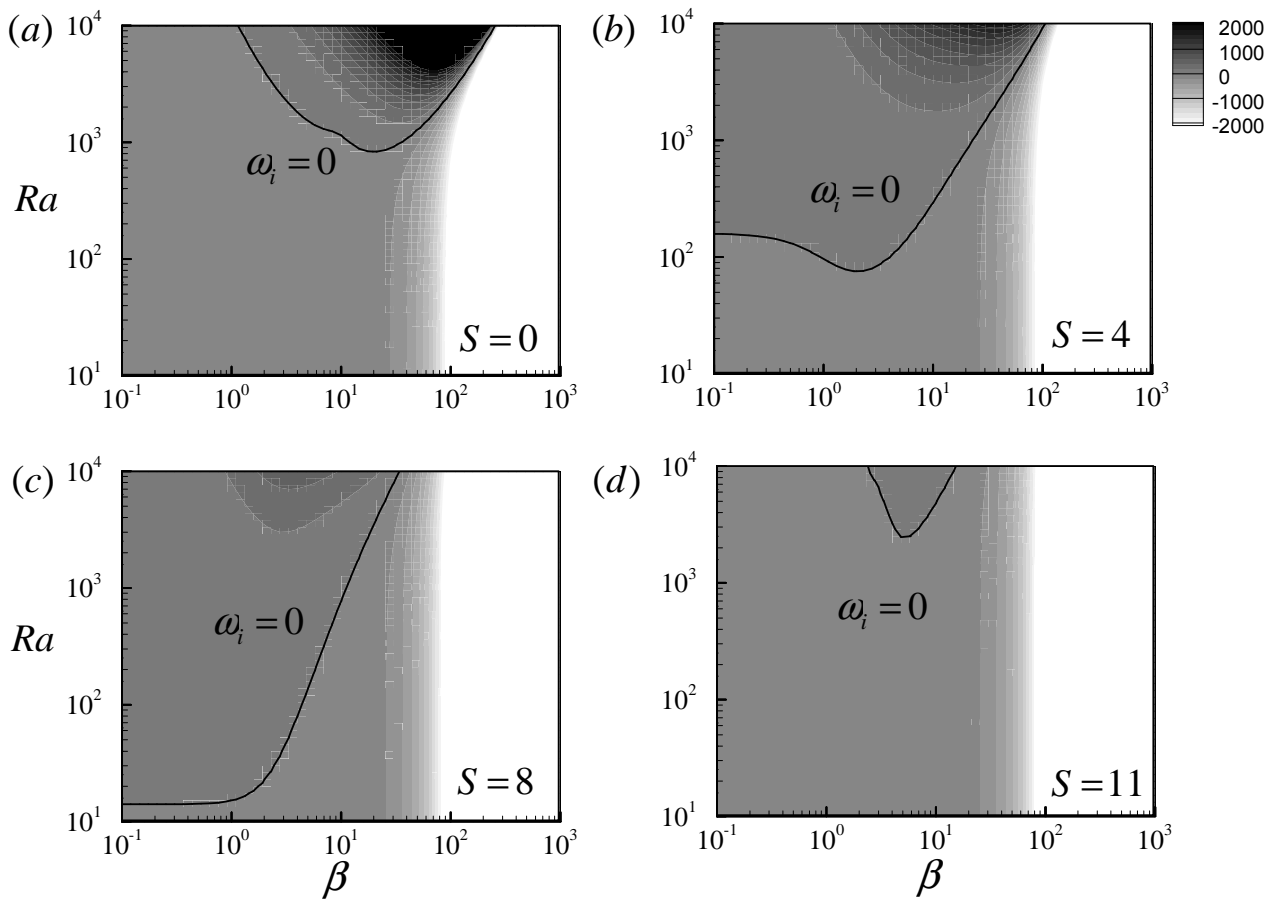

Figure 9. Contour of $\omega_{i}$ of the most unstable mode and its neutral stability curve in the $R a-\beta$ plane for $\alpha=0$ and $\beta \neq 0$ : (a) $S=0$; (b) $S=4$; (c) $S=8$; (d) $S=11$.

and $\lambda_{c}=0.3 \sim 5 \mathrm{~cm}$ in the experiment by Bees \& Hill (1997). In the neutral stability curve for $S=0$, we note that a small kink appears near $\mathrm{Ra}=1000$ and $\alpha=10$. As we shall see in $\S 4.4$, this is due to the appearance of the oscillatory instability, which was also reported in Hill et al. (1989) and Williams \& Bees (2011). For $S=0$, the wavenumber giving the largest growth rate tends to increase with Ra, forming strong instability at $\alpha>20$. However, when shear is introduced, the high-wavenumber instability is drastically damped out (figures $8 b, c$, and $d$ ). In contrast, at relatively low wavenumbers, the shear is destabilising: the suspension is unstable at $\alpha<1 \sim 10$ even for $R a \simeq 10^{3}$ when $S=4$ and $S=8$ (figures $8 b, c$ ). At $S=4$, a kink is present in the neutral stability curve near $\mathrm{Ra} \simeq 500 \sim 700$ and $\alpha \simeq 1 \sim 2$ (figure $8 b$ ), which is caused by a switch between the two most unstable branches with the increase of shear (for the details, see also §4.4). For $S=11$, the instability at small $\alpha$ is stabilised again, thus the suspension does not exhibit instability any more (figure $8 d$ ).

We now consider the streamwise uniform mode $(\alpha=0$ and $\beta \neq 0)$. The contours of the growth rate $\omega_{i}$ with the corresponding neutral stability curve in the Ra- $\beta$ plane are shown in figure 9. For $S=0$, the contour is exactly the same as that in the Ra- $\alpha$ plane (figure $9 a)$. Similarly to the two-dimensional mode $(\alpha \neq 0$ and $\beta=0)$, the addition of shear suppresses instability at high wavenumbers $(\beta>10)$, but it augments the instability at low wavenumbers $(\beta<10$ ) (figures $9 b, c)$. In particular, the streamwise uniform mode shows much stronger destabilisation than the two-dimensional one, resulting in $\mathrm{Ra}_{c} \sim$ $O(10)$ for $S=4$ and $S=8$. The low-wavenumber instability quickly disappears as soon as the shear rate exceeds $S \simeq 10.9$ which gives stably stratified $n_{0}(y)$ (figure $9 d$ ). However, contrary to the two-dimensional mode, the suspension is still linearly unstable 

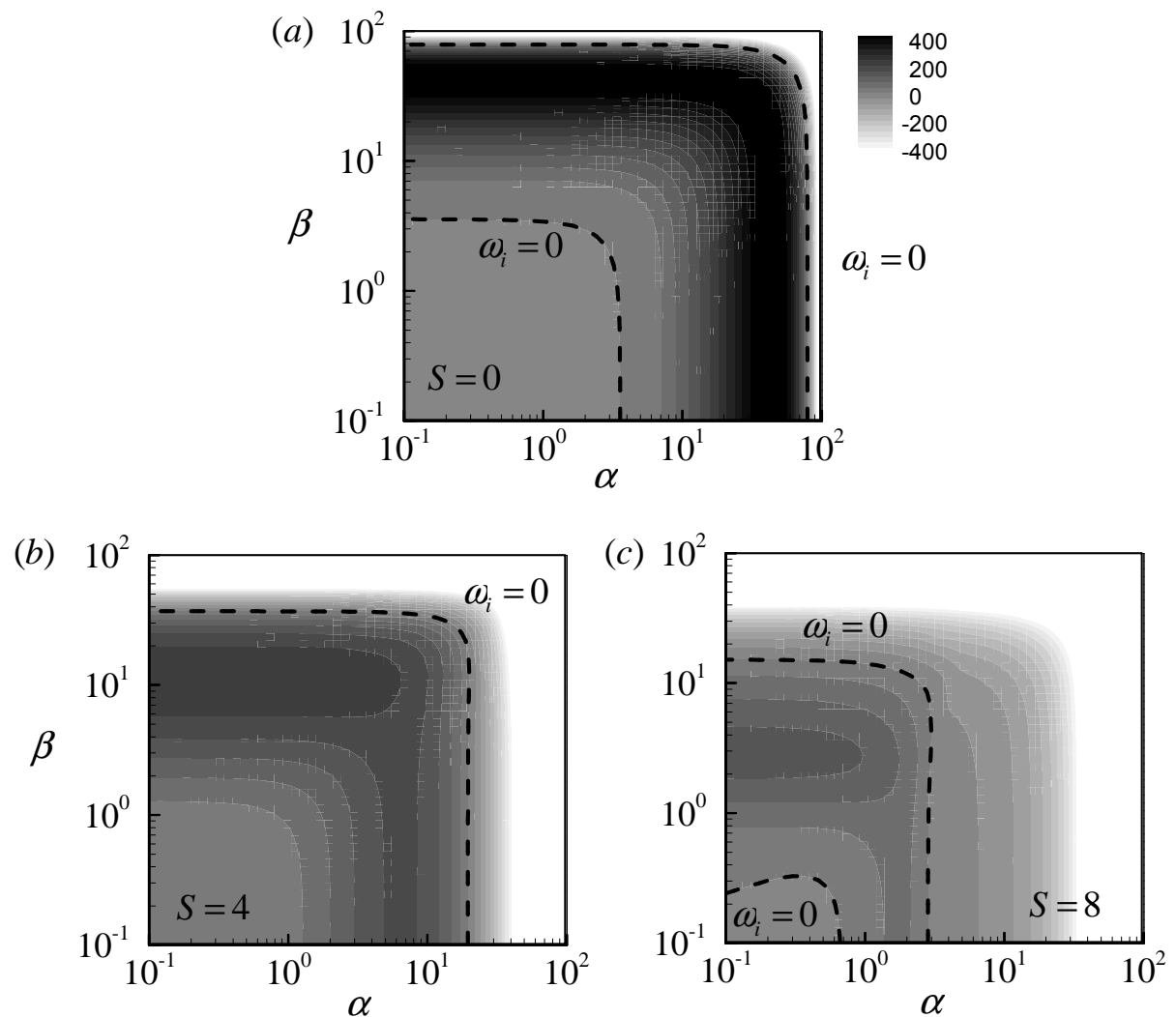

Figure 10. Contour of $\omega_{i}$ of the most unstable mode and its neutral stability curve for the oblique mode $(\alpha \neq 0$ and $\beta \neq 0)$ in the $\alpha$ - $\beta$ plane $(\mathrm{Ra}=2000):(a) S=0 ;(b) S=4 ;(c) S=8$.

at $\beta \simeq 1 \sim 10$, indicating that this instability is not due to the gravitational overturning mechanism.

Finally, oblique modes (i.e. $\alpha \neq 0$ and $\beta \neq 0$ ) are studied. Figure 10 shows the contours of $\omega_{i}$ in the $\alpha-\beta$ plane at $\mathrm{Ra}=2000$. As expected, for $S=0$, the growth rate does not show preference to any specific wavevector directions (figure 10a). With the increase of shear rate (e.g. $S=4), \omega_{i}$ at the high wavenumbers $(\alpha, \beta>10)$ is quickly damped out while that at the low wavenumbers $(\alpha, \beta<10)$ increases a little (figure $10 b)$. The further increase of the shear rate $(S=8)$ stabilises the instability at the low wavenumbers $(\alpha, \beta<10)$, similarly to the two-dimensional and streamwise uniform cases. It is interesting to note that the growth rates for $\beta>\alpha$ are generally larger than those for $\alpha>\beta$ in the presence of shear (figures $10 b$ and $c$ ). In particular, the most unstable set of the two wavenumbers $\alpha$ and $\beta$ appears to be the streamwise uniform mode, indicating that long structures aligned with the shear would appear in the early stages of pattern formation.

\subsection{Eigenfunctions}

Figure 11 visualises the eigenfunction of the most unstable two-dimensional mode for increasing shear rate. For $S=0$, the eigenfunction shows a pair of counter-rotating rolls localised near the top of the domain at which $n_{0}(y)$ is highly concentrated (figure $11 a$ ). The cell number density is largest at $x= \pm 0.314$ and $y \simeq 1$, and this is probably due to the rolls which pump up the cells to this region. For this reason, slightly negative $n^{\prime}$ 

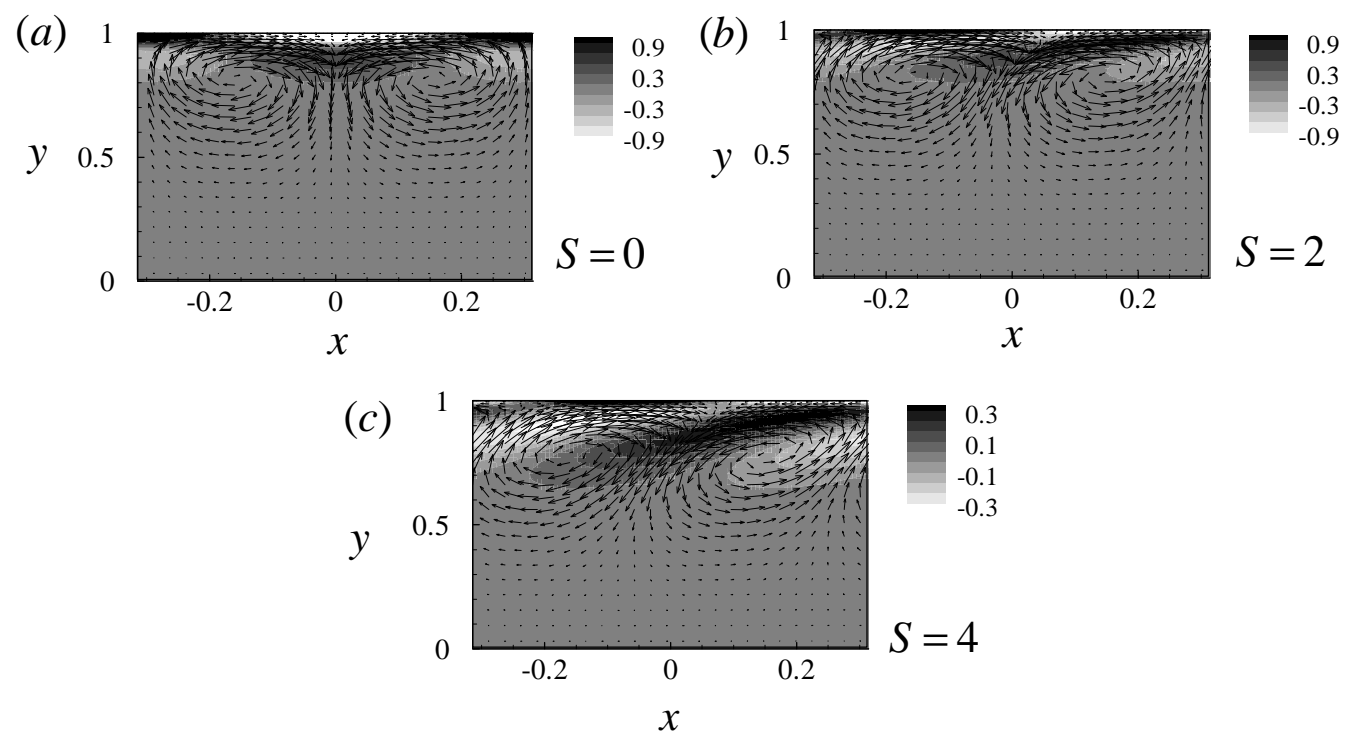

Figure 11. Cross-spanwise view of the eigenfunction for $\alpha=10$ and $\beta=0$ at $\operatorname{Ra}=2000$ : (a) $S=0 ;(b) S=2 ;(c) S=4$. Here, the contours represent the perturbed cell number density and the vectors indicate the streamwise and wall-normal velocities. The flow fields are normalized by $-\max |\hat{v}|_{x=0}$, so that the downwelling region is located along $x=0$. Only the upper half is shown due to highly concentrated nature of the eigenfunction near the upper wall.

seems to appear near $x= \pm 0.314$ and $y \simeq 0.9$, which is located right below the region of the largest cell number density. The cell number density $n^{\prime}$ is smallest at $x=0$ and $y \simeq 1$ probably because the downflow by the rolls takes the cells in this region to the bottom. Therefore, relatively large $n^{\prime}$ also appears at the region right below the location with the smallest cell number density $(x=0$ and $y \simeq 0.9)$. When shear is introduced, the entire structure of the eigenfunction is tilted to the shear direction (figure 11b). This is evidently due to convective transport by the shear, considering the nature of the shear applied. With the increase of the shear rate $S$, the pattern tilts more downstream (figure $11 c)$. It is worth noting that the wall-normal extent occupied by the rolls and the cell number density field appears to increase with the shear rate. This is probably due to the increased thickness of the unstably stratified layer at the top in the basic state: i.e. the decrease of $\kappa$ (figure 7 ).

The most unstable eigenfunction for the spanwise uniform case is visualised in figure 12. In this case, the eigenfunction does not show the tilting with the increase of $S$, as the convective transport by the shear is perpendicular to the $y-z$ plane. The major change by the shear in the eigenfunction structure appears to be the increase of its wall-normal extent. Interestingly, with the increase of the shear rate, the location showing the largest (smallest) perturbed cell number density is spontaneously changed to the region where the downward (upward) velocity of the rolls is large (e.g. $y \simeq 0.7$ in figure $12 c$ ).

\subsection{Branch transition}

Introducing shear also yields some interesting behaviours in the first two most unstable modes. Here, we aim to show the detail of the transition between them as $S$ increases. In $\S 4.2$, we have briefly mentioned that the oscillatory mode $\left(\omega_{r} \neq 0\right)$ appears for small $\alpha$ and $\beta$, giving a small kink in the neutral stability curves for $S=0$ (figures $8 a$ and 9a). This oscillatory mode has also been reported by Hill et al. (1989) and Williams \& 

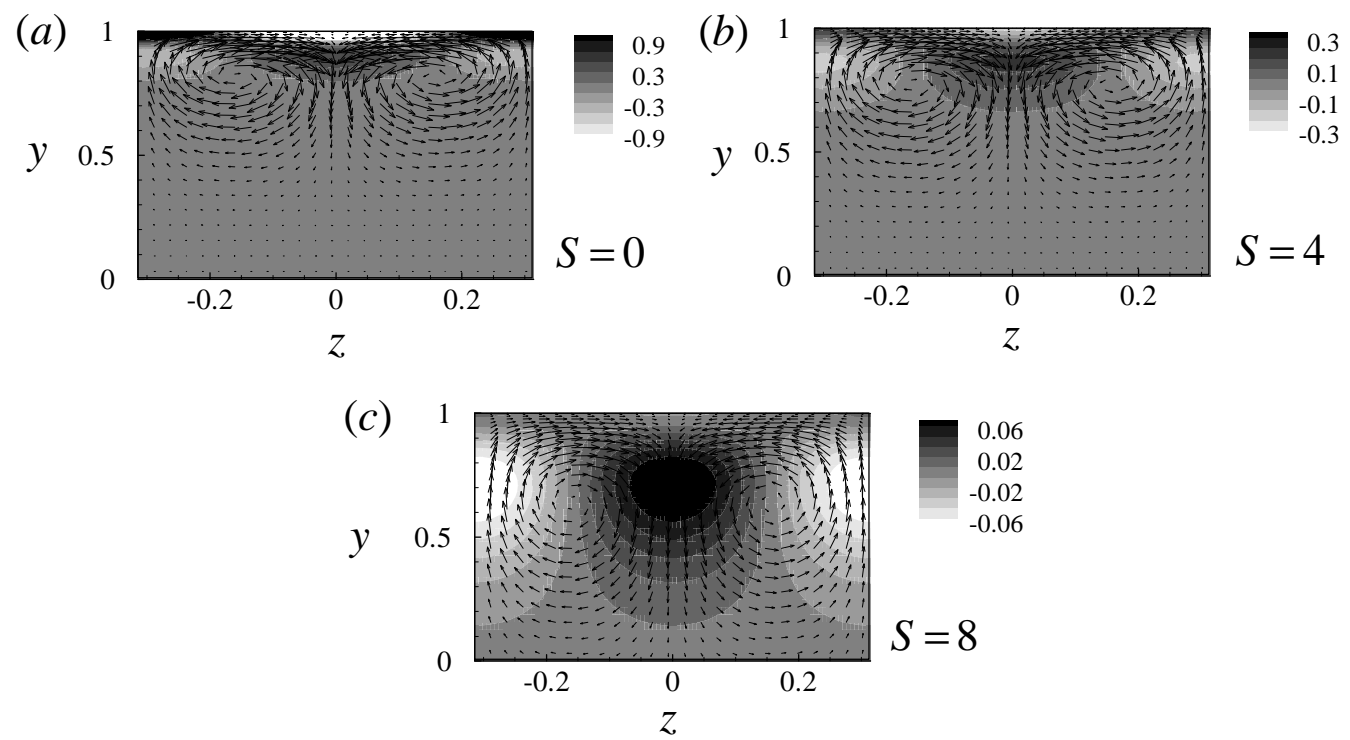

Figure 12. Cross-streamwise view of the eigenfunction for $\alpha=0$ and $\beta=10$ at $\mathrm{Ra}=2000$ : (a) $S=0 ;(b) S=4 ;(c) S=8$. Here, the contour represents the perturbed cell number density and the vectors indicate the spanwise and wall-normal velocities. The flow fields are normalized by $-\max |v(z=0)|$. Only the upper half is shown due to highly concentrated nature of the eigenfunction near the upper wall.
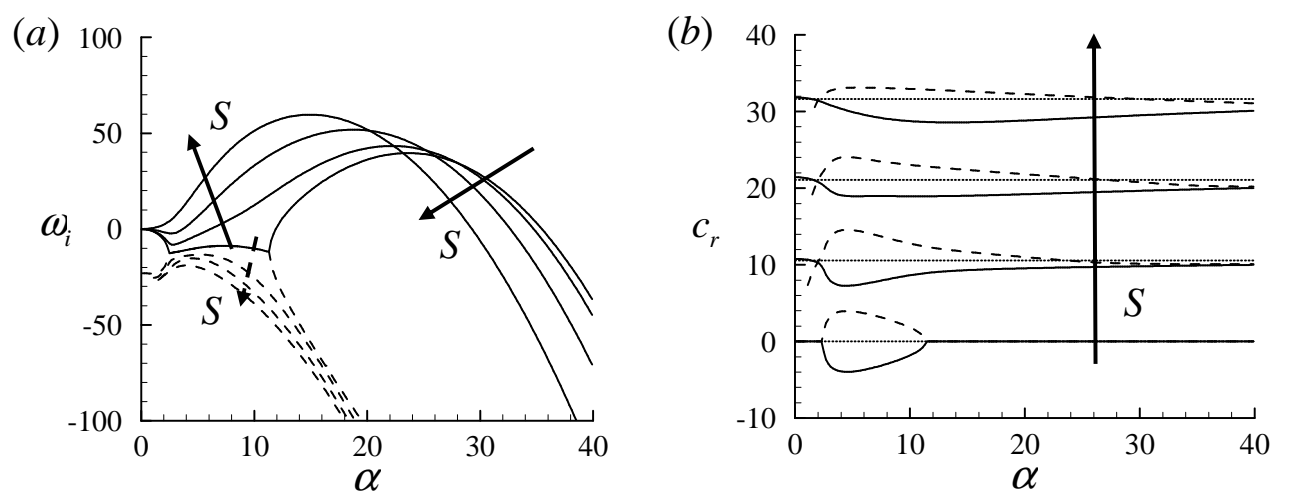

Figure 13. ( $a$ ) Growth rate $\omega_{i}$ and $(b)$ phase velocity $c_{r}\left(=\omega_{r} / \alpha\right)$ of the two-dimensional mode $(\alpha \neq 0$ and $\beta=0)$ for $S=0,0.5,1.0,1.5(\mathrm{Ra}=1000)$ : - , the most unstable mode; - - - the second most unstable mode. In $(b), \cdots \cdots \cdots$ shows the base-flow speed at the upper wall: $U_{\max }=\mathrm{ScRe}$.

Bees (2011), and it has been thought that it originates from the interaction of the cell's gyrotactic nature with the solid boundary in the presence of a roll-like structure (see also $\S 5.3$ for further discussion). Figure 13 shows how the oscillatory modes change with the increase of the shear rate. For $S=0$, the oscillatory behaviour is seen only near $3 \lesssim \alpha \lesssim 12$ where the first two most unstable modes show the same (negative) growth rate (figure $13 a$ ). In particular, one of them propagates upstream whereas the other moves downstream (figure 13b). With introduction of a non-negligible amount of shear, both the modes propagate downstream due to advection by the shear (e.g. $S=0.5$ in figure 13). In this case, the original upstream propagating mode is faster than the speed of the 

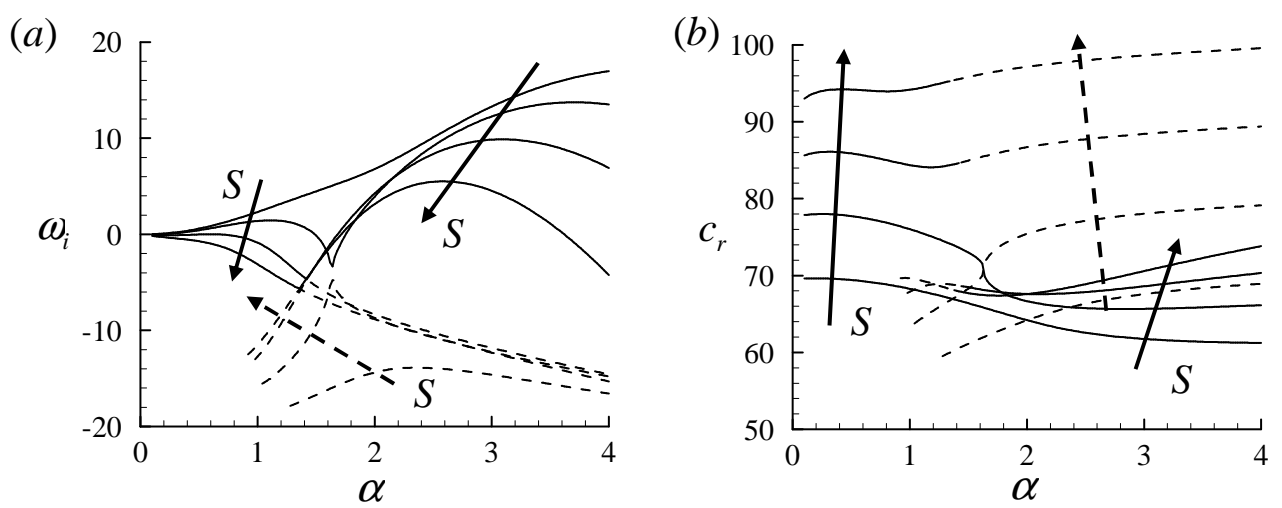

FiguRE 14. (a) Growth rate $\omega_{i}$ and $(b)$ phase velocity $c_{r}\left(=\omega_{r} / \alpha\right)$ of the two-dimensional mode $(\alpha \neq 0$ and $\beta=0)$ near $S=4(\mathrm{Ra}=700):-$, the most unstable mode; - - - - , the second most unstable mode. Here, $S=3.5,4.0,4.5,5.0$.

base flow, whereas the one originally propagating downstream becomes slower (figure $13 b$ ). What is interesting here is that the fast moving mode is stabilised by shear while the slow one is destabilised (figure 13a; see also further discussion in $\S 5.3$ ). This tendency is more pronounced with the increase of $S$ at $\alpha<20 \sim 30$, although the most unstable mode is stabilised at large $\alpha(>30)$.

Further increase of the shear rate yields a branch switching between the first and second most unstable modes. This is seen around $S=4$ at quite small $\alpha(<4)$. We first note that when the shear rate is not very large $(S<2)$, the phase velocity of the first most unstable mode is generally faster than that of the second one for $\alpha<2$ (figure $13 b)$. However, this feature is changed with the further increase of $S$. Figure 14 shows the behaviour of the two most unstable modes at $\mathrm{Ra}=700$ near $S=4$. For the smallest shear rate shown $(S=3.5)$, the growth rates of the two most unstable modes are well separated from each other (figure 14a), and the phase velocity of the first most unstable mode is larger than that of the second one for $\alpha<2$ (figure 14b). When the shear rate is increased to $S=4$, the growth rate curves of the two modes become close to each other particularly near $\alpha \simeq 1.6$ (figure 14a), and the two phase velocity curves exhibit an inflection point at this location (figure 14b). When the shear rate is further increased ( $S=4.5$ ), the first and second most unstable modes at $\alpha \lesssim 2$ turn out to respectively connect to the second and first modes for $\alpha \gtrsim 2$. For the largest shear rate shown $(S=5)$, the two branches are seen to be well separated. The appearance of the kink in the neutral stability curve of the two-dimensional mode for $S=4$ (figure $8 b$ ) is due to this branch switching behaviour.

Finally, transition of the oscillatory branch of the streamwise uniform mode $(\alpha=0$ and $\beta \neq 0)$ is studied. Figure 15 shows the growth rate and the phase velocity of the two most unstable branches with the increase of the shear rate. Contrary to the two-dimensional case in figure 13, the increase of $S$ causes the range of $\beta$ exhibiting oscillatory behaviour to shrink. For sufficiently large shear rates $(S>2)$, the oscillatory behaviour in the two modes disappears and they become completely stationary at all $\beta$ (i.e. $c_{r}=0$ ). In this case, the branch switching behaviour between the two most unstable modes was not observed. 

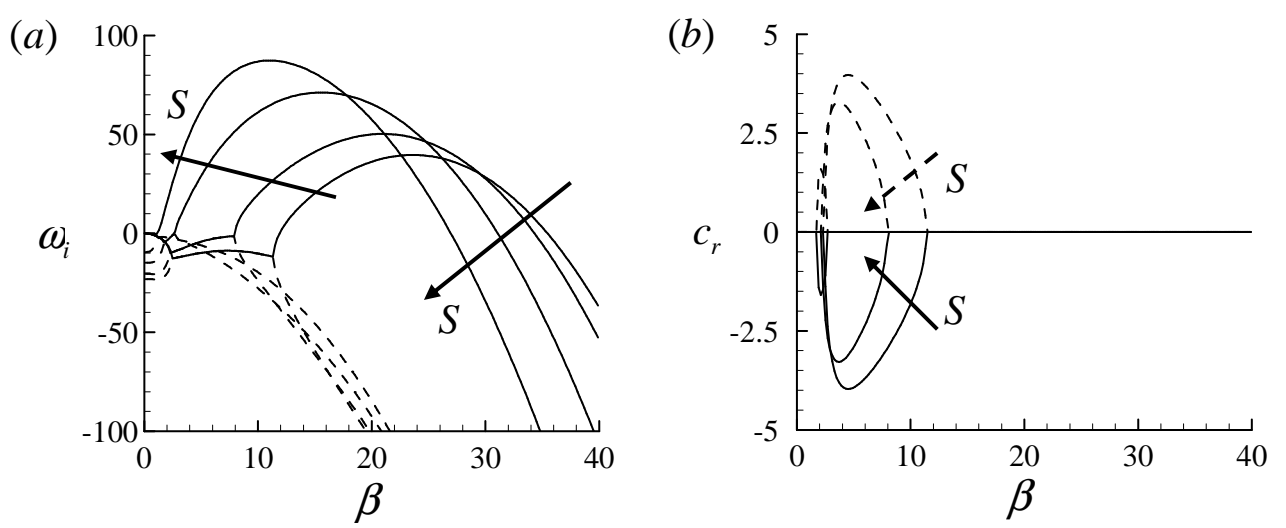

Figure 15. (a) Growth rate $\omega_{i}$ and $(b)$ phase velocity $c_{r}\left(=\omega_{r} / \alpha\right)$ of the streamwise uniform mode $(\alpha=0$ and $\beta \neq 0)$ for $S=0,1,2,3(\mathrm{Ra}=1000)$ : - , the most unstable mode; - - - the second most unstable mode.
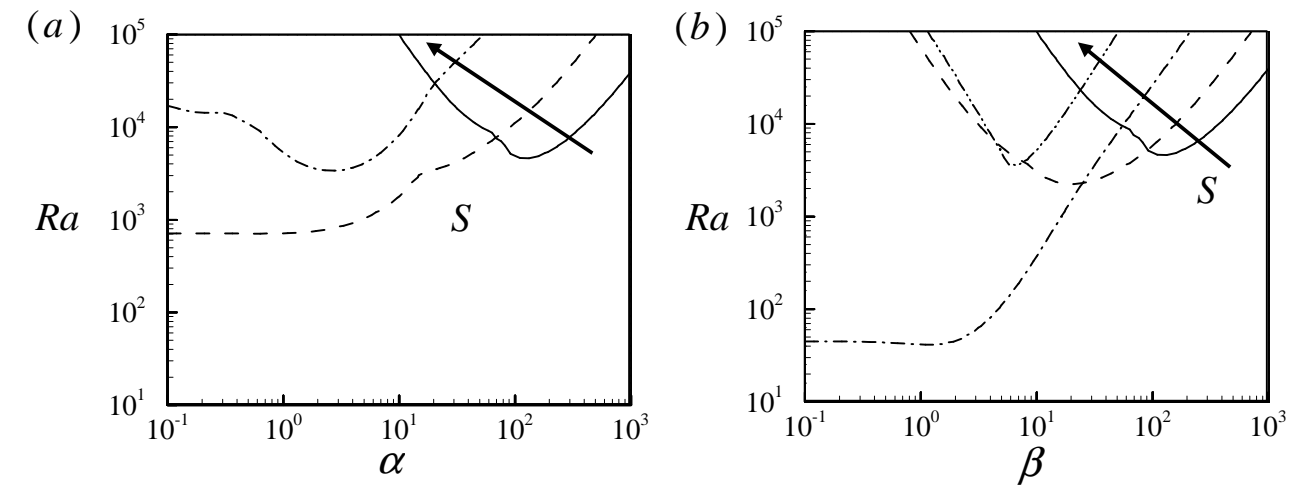

FIgURE 16. Neutral stability curves of the $(a)$ two-dimensional and the $(b)$ streamwise uniform modes $(\tau=1.3 \mathrm{~s}):-, S=0 ;-\cdots, S=4 ; \cdots \cdots, S=8 ; \cdots \cdots, S=11$.

\subsection{Other parameters}

To test the robustness of the present results, some different values of the parameters are also tested. In particular, we focus on studying the effect of the correlation time scale $\tau$, the rotational diffusivity $D_{R}^{*}$, and the depth of suspension $d(=2 h)$. The first two parameters are typically taken from separate experimental measurements (e.g. Hill \& Häder 1997; Vladimirov et al. 2000, 2004; Furlan et al. 2013) and they are unlikely to be constant as shear changes in reality. However, as we shall see here, the change of these parameters does not yield any difference in the qualitative behaviour shown in $\S 4.2$ : for example, in all the cases considered, the instability at high wavenumbers is suppressed by the shear while that at low wavenumbers is destabilised. Also, the streamwise uniform case is found to be most unstable. For this reason, here, we only present the neutral stability curves for the two-dimensional $(\alpha \neq 0$ and $\beta=0)$ and streamwise uniform ( $\alpha=0$ and $\beta \neq 0$ ) modes. Finally, we should remind the reader that $\tau$ appears in the translational diffusivity $D_{V}^{*}$, and hence the dimensionless parameters $S c, R e, D_{T}$ and $D_{R}$ are all inversely proportional to $\tau$ (from $(2.9 \mathrm{~g})$. In fact, the only parameter that does not vary with $\tau$ is $\lambda$. On the other hand, $D_{R}^{*}$ is proportional to $D_{R}$ and $\lambda^{-1}$ only, and $h$ appears in $D_{R}, R a, \alpha$, and $\beta$. 

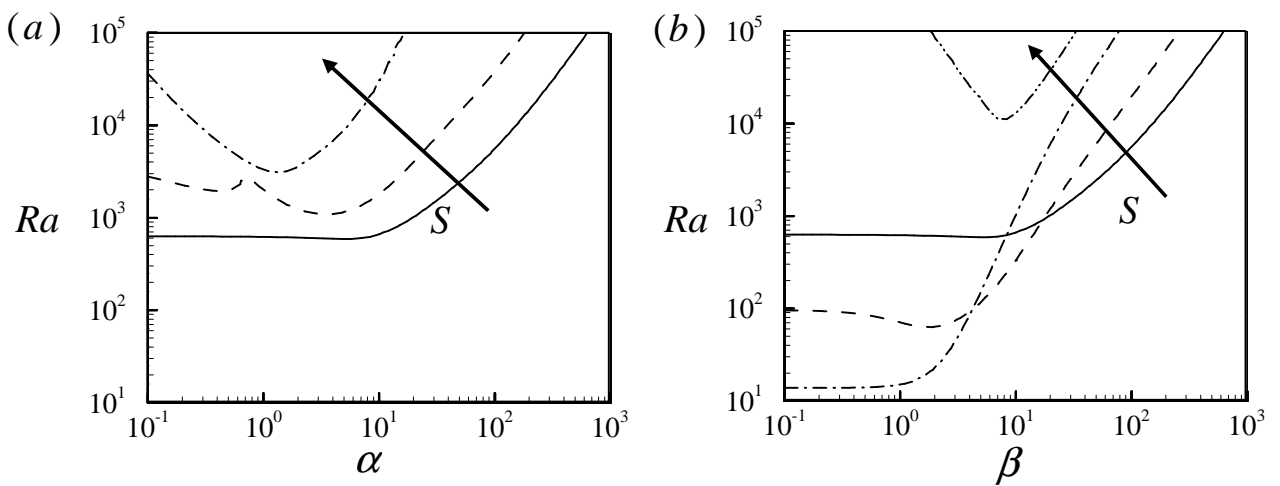

FIgURE 17. Neutral stability curves of the $(a)$ two-dimensional and the $(b)$ streamwise uniform modes $\left(D_{R}^{*}=0.148 \mathrm{sec}, \lambda=2.2\right):-, S=0 ;-\cdot--, S=4 ;-\cdot-\cdot-, S=8 ;-\cdots-\cdots-, S=11$.

Figure 16 shows the neutral stability curves with a different correlation time $\tau=1.3 \mathrm{~s}$, which is about four times smaller than the reference one $\tau=5 \mathrm{~s}$. For $S=0$, the neutral stability curve shows $\mathrm{Ra}_{c} \simeq 3981$ with $\alpha_{c} \simeq 126$. The critical Rayleigh number gives $N_{c} \simeq 1.31 \times 10^{6} \mathrm{cells} / \mathrm{cm}^{3}$, which is not very different from that of the reference case $\left(N_{c} \simeq 1.05 \times 10^{6}\right.$ cells $\left./ \mathrm{cm}^{3}\right)$. This indicates that the increase of $\tau$ does not significantly change the critical cell concentration. In contrast, the critical wavenumber is significantly changed: $\alpha_{c} \simeq 130$ with $\tau=1.3 \mathrm{~s}$. The neutral stability curve suggests that instability at the high wavenumbers is augmented by the decrease of $\tau$. We note that the correlation time scale $\tau$ is the parameter that directly controls translational diffusivity. Therefore, its decrease reduces the effect of translational diffusion of the system, leading to the appearance of such an instability at high wavenumbers. However, the behaviour of the neutral curves with the increase of the shear rate does not show any qualitative difference from that in the reference case (compare figure 16 with figures 8 and 9).

A different rotational diffusivity $D_{R}^{*}=0.148 \mathrm{~s}$, which is larger than the reference one, is also tested while keeping $\lambda\left(=1 / 2 B D_{R}^{*}\right)=2.2$. The decrease of the rotational diffusivity with the same $\lambda$ only yields the decrease of two dimensionless parameters: i.e. $G_{1}(=0.17)$ and $G_{2}(=0.022)$. Figure 17 shows the neutral stability curves for the two-dimensional and streamwise uniform modes. For $S=0$, the critical Rayleigh number is lowered a little $\left(\mathrm{Ra}_{c} \simeq 575\right)$ giving $N_{c} \simeq 7.3 \times 10^{5} \mathrm{cells} / \mathrm{cm}^{3}$. However, a quite low critical wavenumber is obtained $\left(\alpha_{c} \simeq 5\right)$. We note that the oscillatory branch does not appear in this case (see also $\$ 5.3$ below). As mentioned, the change in the neutral stability curves with the shear rate $S$ is found to be qualitatively the same as that of the reference case (compare figure 17 with figures 8 and 9).

Finally, we consider a smaller depth $(d=0.05 \mathrm{~cm})$. The decrease of the depth increases the effect of translational diffusion of the linear system (2.22), leading to smaller $\kappa(=2.41)$ (see figure 7). The decrease of the depth significantly lowers the critical Rayleigh number $\left(\mathrm{Ra}_{c} \simeq 9.2\right)$, consistent with the findings by Bees \& Hill (1998). The critical wavenumber is obtained as $\alpha_{c} \simeq 2.5$, much smaller than that of the reference case. The critical cell concentration and wavelength are found respectively as $N_{c} \simeq 1.2 \times 10^{7} \mathrm{cells} / \mathrm{cm}^{3}$ and $\lambda_{c} \simeq 0.06 \mathrm{~cm}$. This suggests that the decrease of the depth increases the critical cell concentration whereas it decreases the actual pattern length that we would observe experimentally despite the decrease of the dimensionless critical wavenumber $\alpha_{c}$ (i.e. the increase of the dimensionless critical wavelength). We should also point out that this behaviour is consistent with the experimental observation in Bees \& Hill (1997). Similarly 

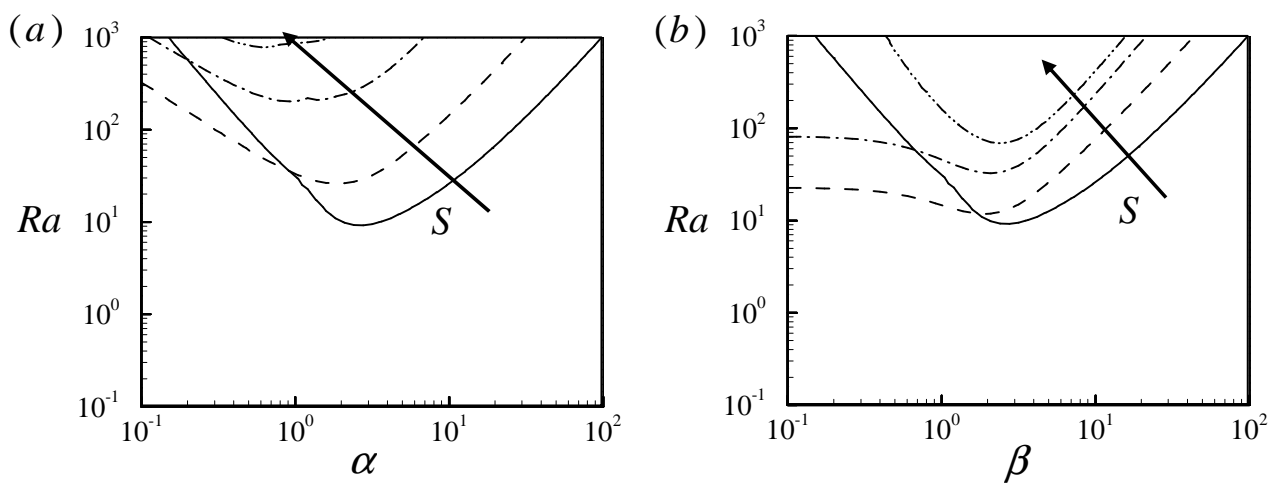

FIgURE 18. Neutral stability curves of the $(a)$ two-dimensional and the $(b)$ streamwise uniform modes $(d=0.05 \mathrm{~cm}):-, S=0 ;----, S=4 ;-\cdot-\cdot, S=8 ;-\cdots \cdots, S=11$.

to the previous cases, the increase of the shear rate yields qualitatively the same change in the neutral curves (compare figure 18 with figures 8 and 9). However, interestingly, both the two-dimensional and the streamwise uniform modes show that the amount of destabilisation at low wavenumbers is considerably smaller than with the large depth (e.g. $d=0.5 \mathrm{~cm})$.

\section{Discussion}

Thus far, we have studied how uniform shear affects the linear stability in shallow layers of a gyrotactic microorganism suspension. The shear in the suspension is found to suppress instability at high wavenumbers, whereas it is destabilising at low wavenumbers. The most unstable mode is found to be a pair of counter-rotating rolls, that are uniform and aligned with the shear (i.e. the streamwise unform mode). For very large shear rates (e.g. $S>11$ ), the instability is found to be strongly damped out. We should emphasise that these are robust features because they are not qualitatively changed by variation of the parameters. This suggests that the behaviour is a robust consequence of specific physical mechanisms in the system.

\subsection{Physical mechanisms of bioconvective instability revisited}

Before we investigate the role of shear, it is helpful to examine the case in the absence of shear. Using $\zeta_{2}=\zeta_{6}=0$ (figure 6 ) and neglecting $\partial / \partial z$ due to the horizontal isotropicity, the equation for $n^{\prime}(2.18 c)$ with $S=0$ is given as follows:

$$
\frac{\partial n^{\prime}}{\partial t}+L_{a d} n^{\prime}+v^{\prime} \frac{d n_{0}}{d y}+G_{1} \zeta_{1} n_{0} \frac{\partial \omega_{3}^{\prime}}{\partial x}-G_{2} \zeta_{5} \frac{d n_{0}}{d y} \frac{\partial \omega_{3}^{\prime}}{\partial x}=0,
$$

where

$$
\begin{aligned}
L_{a d} & =\operatorname{ScRe} U \frac{\partial}{\partial x}+V_{c}\left\langle e_{1}\right\rangle_{0} \frac{\partial}{\partial x}+\left(V_{c}\left\langle e_{2}\right\rangle_{0}-V_{s}\right) \frac{\partial}{\partial y} \\
& -D_{T 0}^{11} \frac{\partial^{2}}{\partial x^{2}}-2 D_{T 0}^{12} \frac{\partial^{2}}{\partial x \partial y}-D_{T 0}^{22} \frac{\partial^{2}}{\partial y^{2}} .
\end{aligned}
$$

Here, the $L_{a d}$ operator represents the advection and diffusion, which do not give instability. Therefore, the source terms for instability turn out to be the last three terms in the left-hand side of $(5.1 a)$. The first one describes the instability due to the unstable stratification interacting with the velocity perturbation, which appears in the equations 


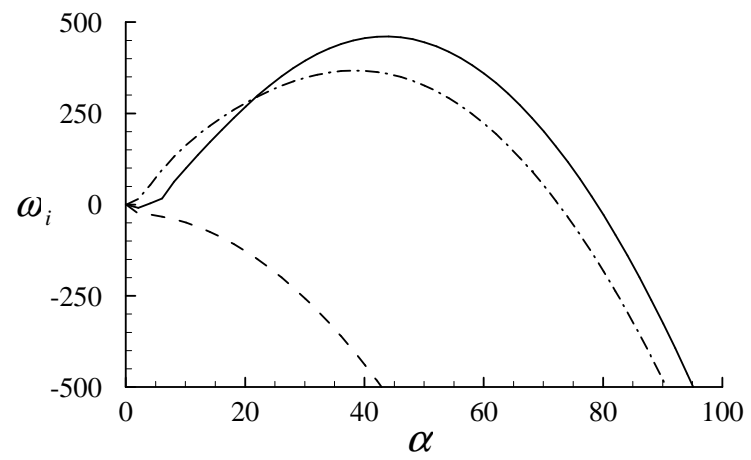

FiguRE 19. Growth rate $\omega_{i}$ with respect to $\alpha(\mathrm{Ra}=2000$ and $S=0):-$, with all the terms in $(5.2 a) ;----$, without $n_{0}\left[\kappa+\chi_{1} \alpha^{2}\right] \hat{v} ;-\cdot-\cdot-$, without $n_{0} \chi_{1} \mathcal{D}^{2} \hat{v}$.

for the classical Rayleigh-Bénard convection (Drazin \& Reid 1981). In this respect, this term represents true gravitational overturning. The second one with $G_{1}$ is proportional to $n_{0}(y)$, and thus it can be active even in uniform suspensions. Therefore, this term is the source term of the gyrotactic instability (Pedley et al. 1988): it actually describes the horizontal flux of the swimming vector $\left(V_{c}\left\langle e_{1}\right\rangle^{\prime}\right)$ by the spanwise vortical perturbation $\omega_{3}^{\prime}$. The last term with $G_{2}$ can also give instability, as we shall see. We note that $\zeta_{5}$ here is actually from $D_{T}^{12^{\prime}}$ (see $(2.17 b)$ ), implying that this term is associated with the cross diffusion flux created by the vortical perturbation $\omega_{3}^{\prime}$.

The last instability mechanism mentioned here is not new because it also appears in the equations in Bees \& Hill (1998). However, it has not been fully discussed although it describes a physical process essentially different from that of the gyrotactic instability (e.g. Pedley et al. 1988; Pedley \& Kessler 1990). Similarly to the gyrotactic instability described in the Introduction, let us suppose there is a small blob of high cell concentration. Let us also suppose the presence of the unstable stratification in the surroundings as the term actually includes $d n_{0} / d y$. Since the blob is denser than the surroundings, it will sink and create downflow behind it. The downflow appears to introduce a vortical perturbation, thus it induces negative cross-diffusion flux owing to the gyrotactic behaviour of the cells (see $D_{T 0}^{12}$ in figure 3 ). In the presence of the unstable stratification, the cross-diffusion flux is not negligible and is directed from the less dense surroundings to the denser blob and its wake. This mechanism will make the blob denser still and create a more rapid downflow, similarly to the gyrotactic instability mechanism. It should be mentioned that this instability mechanism also essentially originates from the gyrotactic nature of the cell. Therefore, it would be appropriate to interpret it as a different mechanism of gyrotactic instability. We finally note that this instability mechanism may depend on the choice of the translational diffusivity model (2.6). However, the negative cross diffusivity induced by the shear has also been observed in other diffusivity models: for example, in the generalised Taylor dispersion theory (Bearon et al. 2012). Therefore, the mechanism would probably be a robust instability mechanism for a real system. Hereafter, we shall call this instability the diffusion-oriented instability to distinguish it from the original gyrotactic instability mechanism in Pedley et al. (1988).

Further examination of (5.1) gives more physical insight. Consider a single FourierLaplace mode (e.g. $\left.n^{\prime}(x, y, t)=\hat{n}(y, t) e^{i(\alpha x-\omega t)}\right)$. Then, $\partial \omega_{3}^{\prime} / \partial x$ in $(5.1 a)$ becomes $-\left(\alpha^{2}-\right.$ $\left.\mathcal{D}^{2}\right) \hat{v}$ (see (2.20) and (2.21)). Also, $d n_{0} / d y=\kappa n_{0}$ and thus $(5.1 a)$ becomes

$$
-i \omega \hat{n}+\left.L_{C}\right|_{\beta=0} \hat{n}+n_{0}\left[\kappa+\chi_{1}\left(\alpha^{2}-\mathcal{D}^{2}\right)\right] \hat{v}=0,
$$


where

$$
\chi_{1}=-G_{1} \zeta_{1}+\kappa G_{2} \zeta_{5} .
$$

Here, the operator $L_{C}$ is given in $(2.22 f)$. A numerical examination reveals that the effect of the term with $\mathcal{D}^{2}$ is not significant for instability (see figure 19). Therefore, the instability would appear if the following parameter is positive and sufficiently large:

$$
\eta=\kappa+\alpha^{2} \chi_{1}
$$

Here, the first term represents the contribution of the gravitational overturning, while the second represents the contribution of the gyrotactic and the diffusion-oriented mechanisms. It is interesting to note that both the gyrotactic and the diffusion-oriented mechanisms actually behave in the same way as gravitational overturning. Since the gravitational instability appears for $\kappa>0$, both the mechanisms would also be active as sources of instability if $\zeta_{1}<0$ and $\zeta_{5}>0$ for $\kappa>0$. For $S=0, \zeta_{1}=-0.103$ and $\zeta_{5}=0.023$ with $\kappa>0$, indicating that the two mechanisms are indeed involved in the instability generation. Furthermore, $-G_{1} \zeta_{1}=0.039$ and $\kappa G_{2} \zeta_{2}=0.026$ in $(5.2 b)$, implying that they are of comparable importance.

Equation (5.3) also suggests that the gravitational mechanism would be the only important one at small $\alpha$ while the gyrotactic and diffusion-oriented mechanisms would play a dominant role at large $\alpha$. The streamwise wavenumber, above which the gyrotactic and diffusion-oriented mechanisms would begin to dominate over the gravitational one, is thus roughly given by

$$
\alpha_{b}=\sqrt{\left|\kappa / \chi_{1}\right|}
$$

For the reference parameters, $\alpha_{b} \simeq 19$, implying that the high-wavenumber instability for $\alpha>\alpha_{b}$ in figure $8(a)$ is mostly due to the gyrotactic and diffusion-oriented mechanisms. It should also be noted that $\chi_{1} \sim O\left(h^{-1}\right)$ while $\kappa \sim O(h)$, suggesting that the role of the gravitational mechanism would be diminishingly small in very shallow suspensions.

In support of these arguments, we perform a set of numerical experiments in which we artificially suppress one or two of the instability mechanisms. Figure 20 shows the results of the numerical experiment. In each case, we consider a Rayleigh number which is sufficiently large compared with the critical value that all the instability mechanisms would be active. For the reference parameters (figure 20a), suppression of the gravitational term $\left(v^{\prime} d n_{0} / d y\right)$ leads to lack of the growth rate at the wavenumbers below $\alpha_{b}=19$. On the other hand, suppression of either the gyrotactic $\left(G_{1} \zeta_{1} n_{0} \partial \omega_{3} / \partial x\right)$ or the diffusionoriented instability $\left(G_{2} \zeta_{1} d n_{0} / d y \partial \omega_{3} / \partial x\right)$ terms gives a significant amount of reduction in the growth rate for $\alpha>\alpha_{b}$. A similar experiment is conducted with a smaller correlation time $(\tau=1.3)$ (figure 20b). The reduced $\tau$ basically decreases the role of the translational diffusion, thereby yielding a large value of $\kappa=93$ and $\alpha_{b}=38$. For the same reason, the reduced $\tau$ reveals instability at fairly high wavenumbers $(100<\alpha<350)$. In this range of the wavenumber, the gravitational mechanism is supposed to be quite weak according to $(5.4)$. This is consistent with the results in figure $20(b)$ : the inhibition of the gravitational term yields only little change. However, the inhibition of either the gyrotactic or the diffusion-oriented instability terms significantly reduces the growth rate, confirming their dominance at the high wavenumbers. The effect of rotational diffusivity is also tested with $D_{R}^{*}=0.148 \mathrm{~s}$, which is larger than the reference value (figure $20 \mathrm{c}$ ). The increase of $D_{R}^{*}$ reduces both $G_{1}$ and $G_{2}$ (see $(2.18 d)$ ), giving a lower $\chi_{1}=0.030$ and $\alpha_{b}=28.6$. Numerical experiments show that the role of the gyrotactic and diffusion-oriented instability mechanisms is indeed reduced, thereby leading to a significant reduction in the growth rate in response to the suppression of the gravitational mechanism. Finally, the effect of the suspension depth is examined (figure $20 d$ ). The decrease of the depth yields $\kappa=2.41$ 

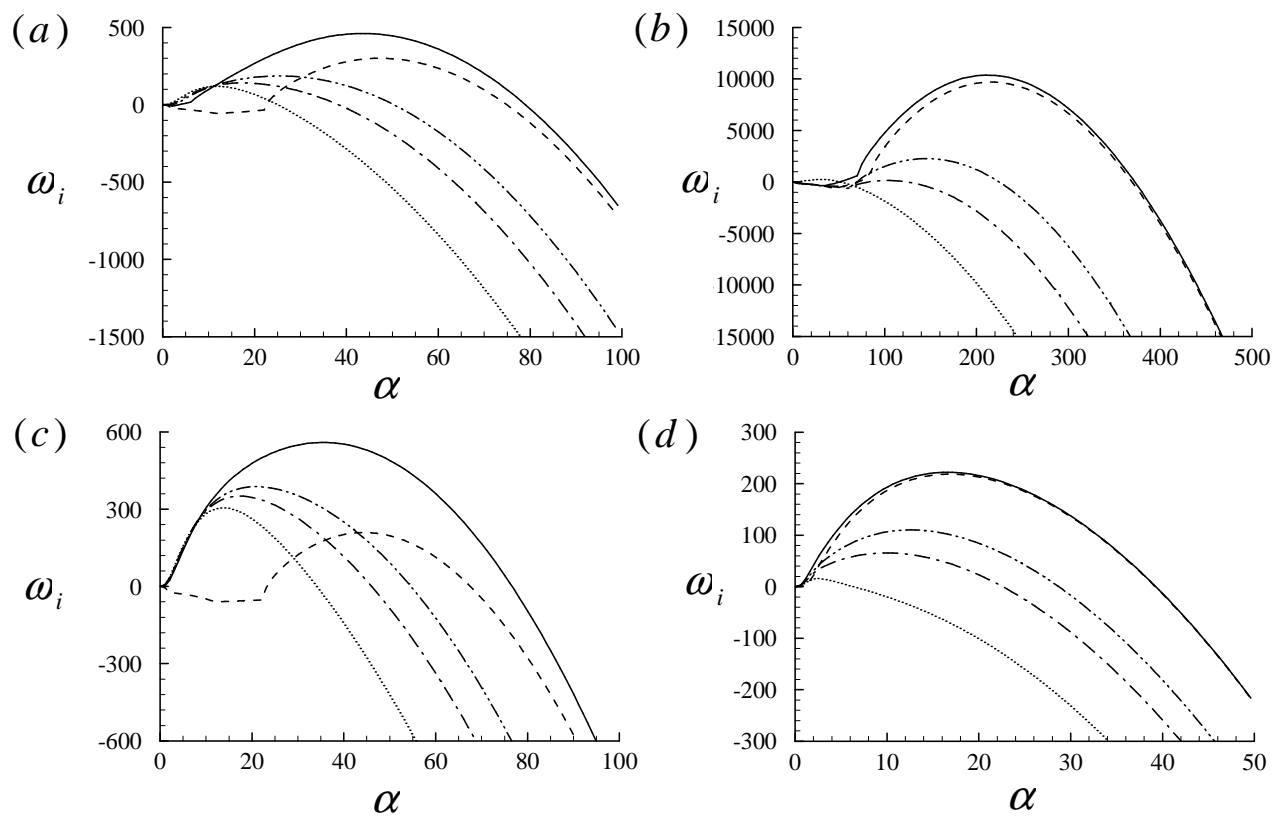

FIGURE 20. Numerical experiments with artificial suppression of each source term of instability: (a) the reference parameters in table $1(\mathrm{Ra}=2000) ;(b) \tau=1.3 \mathrm{~s}(\mathrm{Ra}=10000) ;(c) D_{R}^{*}=0.148 \mathrm{~s}$ with $\lambda=2.2(\mathrm{Ra}=5000),(d) h=0.05 \mathrm{~cm}(\mathrm{Ra}=100)$. Here, - , with full terms in (5.1); ---- , without the third term $\left(v^{\prime} d n_{0} / d y\right) ;-\cdot-\cdot-$, without the fourth term $\left(G_{1}=0\right) ;-\cdots-\cdots-$, without the fifth term (i.e. $\left.G_{2}=0\right) ; \cdots \cdots \cdots$, without the fourth and fifth terms $\left(G_{1}=G_{2}=0\right)$.

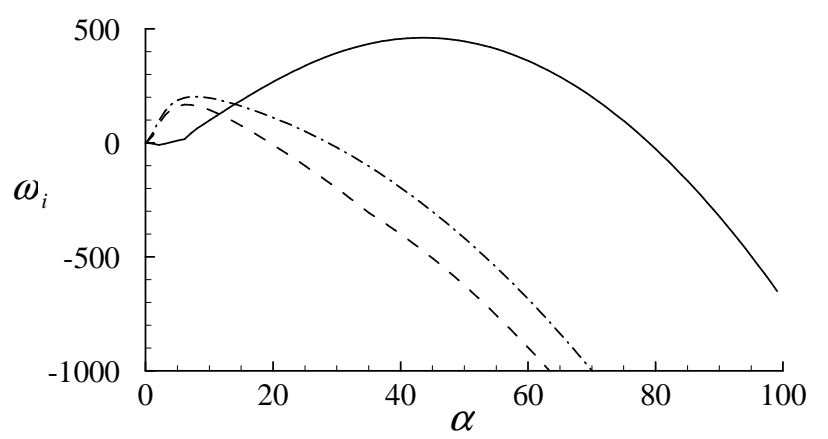

FiguRE 21. Growth rate $\omega_{i}$ with respect to $\alpha:-, S=0 ;----, S=4 ;-\cdot-\cdot-, S=4$ with $\chi_{2}=0$.

and $\chi_{1}=0.65$, giving $\alpha_{b}=1.9$. This suggests that the gravitational mechanism would be important only for quite small $\alpha$. Indeed, numerical experiment shows that the gyrotactic and the diffusion-oriented instability mechanisms dominate over the gravitational overturning at most of the wavenumbers.

\subsection{Stabilisation by shear - the reduced gyrotactic response and advective transport by the base flow}

Now, we see how the shear plays a stabilising role in bioconvective instability. Neglecting the terms with $\hat{\eta}$, the equation for $\hat{n}$ in the two-dimensional mode $(\alpha \neq 0$ and $\beta=0)$ is 

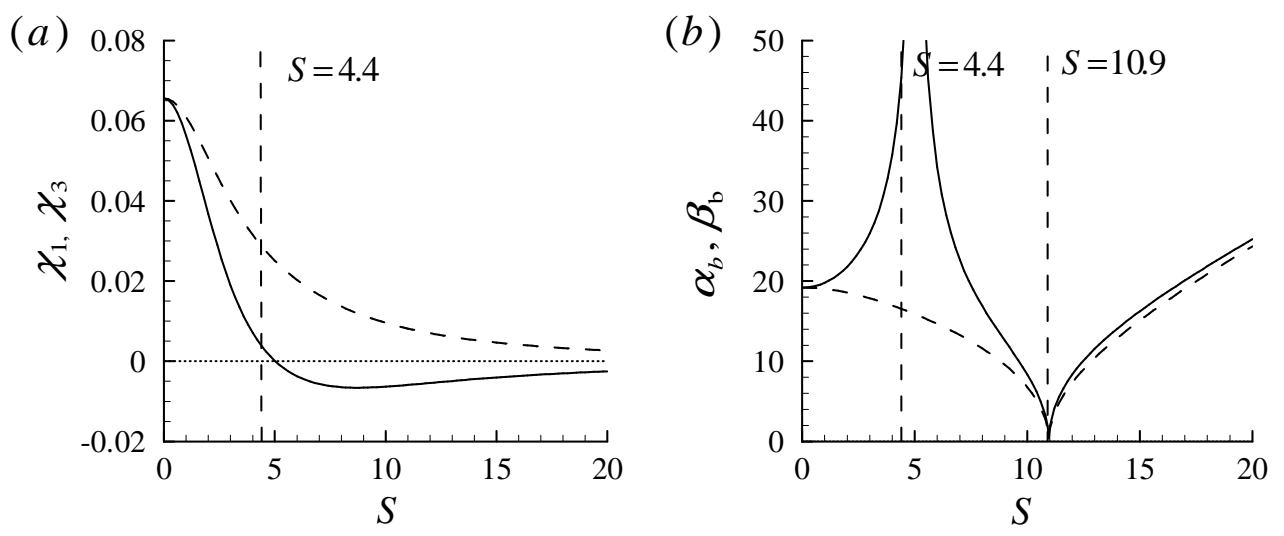

Figure 22. Variation of $(a) \chi_{1}(-)$ and $\chi_{3}(----)$, and $(b) \alpha_{b}\left(=\sqrt{\left|\kappa / \chi_{1}\right|}\right)(-)$ and $\beta_{b}\left(=\sqrt{\left|\kappa / \chi_{3}\right|}\right)(----)$ with the shear rate $S$.

written in the following form:

$$
\begin{gathered}
-i \omega \hat{n}+\left.L_{C}\right|_{\beta=0} \hat{n}+n_{0}\left[\kappa+\chi_{1}\left(\alpha^{2}-\mathcal{D}^{2}\right)\right] \hat{v}+\frac{i \chi_{2}}{\alpha} \mathcal{D}\left[n_{0}\left(\alpha^{2}-\mathcal{D}^{2}\right) \hat{v}\right]=0, \\
\chi_{2}=G_{1} \zeta_{2}-G_{2} \kappa \zeta_{6} .
\end{gathered}
$$

Similarly, the equation for $\hat{n}$ in the streamwise uniform mode $(\alpha=0$ and $\beta \neq 0)$ is given as

$$
\begin{gathered}
-i \omega \hat{n}+\left.L_{C}\right|_{\alpha=0} \hat{n}+n_{0}\left[\kappa+\chi_{3}\left(\beta^{2}-\mathcal{D}^{2}\right)\right] \hat{v}=0, \\
\chi_{3}=G_{1} \zeta_{3}-G_{2} \kappa \zeta_{7} .
\end{gathered}
$$

Compared to $(5.2 a),(5.5 a)$ now shows an additional term with $\chi_{2}$ due to the non-zero $\zeta_{2}$ and $\zeta_{6}$. This term originates from the wall-normal transport by the perturbed swimming vector and diffusivity tensor: i.e. $\partial / \partial y\left(V_{c}\left\langle e_{2}\right\rangle^{\prime} n_{0}-D_{T}^{22^{\prime}} d n_{0} / d y\right)$ in $(2.14 f)$. A numerical examination reveals that the role of this term is limited in instability generation (see figure 21), allowing us to neglect it. Also, as shown in figure $19, \mathcal{D}^{2}$ in the third term of $(5.5 a)$ can also be neglected. Then, (5.5) is approximated as

$$
-i \omega \hat{n}+\left.L_{C}\right|_{\beta=0} \hat{n}+n_{0}\left(\kappa+\chi_{1} \alpha^{2}\right) \hat{v} \simeq 0
$$

Similarly, (5.6) becomes

$$
-i \omega \hat{n}+\left.L_{C}\right|_{\alpha=0} \hat{n}+n_{0}\left(\kappa+\chi_{3} \beta^{2}\right) \hat{v} \simeq 0 .
$$

These approximate equations suggest that $\chi_{1}$ and $\chi_{3}$ play a crucial role in regulating instability. However, we also note that $\left.L_{C}\right|_{\beta=0}$ now contains the convective transport term $i \alpha \operatorname{ScRe} U$. As we shall see, this term also plays a key role in suppressing instability for sufficiently large $S$.

We first see how $\chi_{1}$ and $\chi_{3}$ change with the increase of the shear rate (see figure $22 a$ ): both parameters decrease with the increase of $S$, which explains the disappearance of the instability at high wavenumbers (e.g. figures $8 b$ and $9 b$ ). In particular, $\chi_{1}$ is found to decay faster than $\chi_{3}$, even becoming negative at $S \simeq 5$. The negative $\chi_{1}$ implies that the term with $\chi_{1}$ can actually stabilise the instability for $S>5$, and this is indeed confirmed numerically (not shown). It is worth noting that $S=5$ is not very far from $S_{c}=4.4$ at which a deterministic cell would begin to tumble. The faster decay of $\chi_{1}$ explains why the streamwise uniform mode is more unstable than the two-dimensional one at relatively 


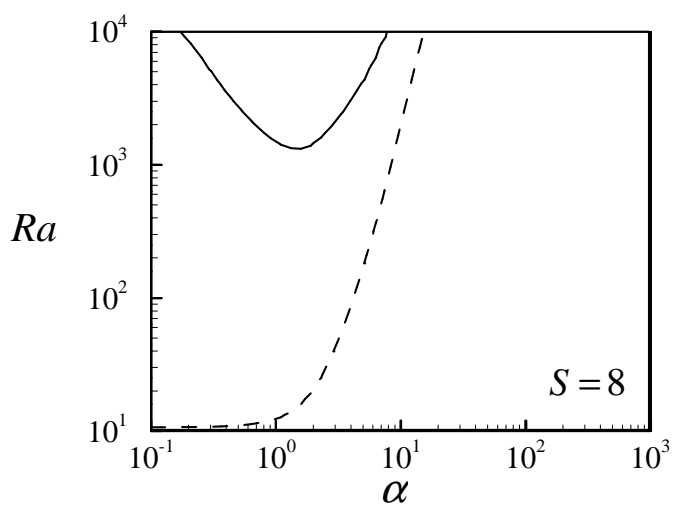

FiguRE 23. Neutral stability curves for $\alpha \neq 0$ and $\beta=0$ with (_- ) and without (- - - - ) the convective transport term.

high wavenumbers (figures $10 b$ ). We note that the behaviour of $\chi_{1}$ and $\chi_{3}$ with the shear rate $S$ is essentially due to $\zeta_{i}$, which describes the gyrotactic response of the mean cell swimming direction $\langle\mathbf{e}\rangle^{\prime}$ and the diffusivity tensor $\mathbf{D}_{T}^{\prime}$ (see figure 6). Interestingly, the quantities related to the diffusivity tensor $\left(\zeta_{5}\right.$ and $\left.\zeta_{7}\right)$ are generally seen to decay faster than those related to the mean cell swimming direction $\left(\zeta_{1}\right.$ and $\left.\zeta_{3}\right)$. This implies that the shear suppresses the diffusion-oriented instability mechanism more effectively than the gyrotactic instability mechanism.

The behaviour of the wavenumber that gives the boundary between the regimes of the gravitational-overturning and the gyrotactic-response dominance is also studied. We start by defining the boundary wavenumber for the streamwise uniform mode as $\beta_{b}=\sqrt{\left|\kappa / \chi_{3}\right|}$. Figure $22(b)$ shows the behaviour of both $\alpha_{b}$ and $\beta_{b}$ with the shear rate. For small $S(<5)$, $\alpha_{b}$ quickly increases and diverges to $\alpha_{b}=\infty$ at $S \simeq 5$ because $\chi_{1}$ quickly reaches zero at this shear rate. On the other hand, $\beta_{b}$ decreases slowly with the increase of $S$. For $S>5$, $\alpha_{b}$ falls dramatically until $S \simeq 11$, at which $\kappa=0$. However, $\beta_{b}$ continue to decay slowly as that for $S<5$. We note that, in this range of $S, \chi_{1}<0$ unlike $\chi_{3}$. Therefore, the instability of the two-dimensional mode at $\alpha>\alpha_{b}$ is supposed to be strongly damped, and this is indeed seen in the neutral stability curve for $S=8$ (figure $8 c$ ). For $S \geqslant 11$, both $\alpha_{b}$ and $\beta_{b}$ gradually increase with $S$. In this case, the gravitational mechanism does not give instability any more because $n_{0}(y)$ is stably stratified (figure 7 ). Therefore, the two-dimensional mode is supposed to be stable, consistent with the neutral stability curve given in figure $8(d)$. However, even in this range, $\chi_{3}>0$, indicating that the streamwise uniform mode can bear instability for $\beta>\beta_{b}$ by the gyrotactic and the diffusion-oriented mechanisms (note that $\zeta_{3}>0$ and $\zeta_{7}<0$ ). This explains why the streamwise uniform mode is unstable even for $S \simeq 11$ (figure $9 d$ ).

In Rayleigh-Bénard convection, introducing shear has been shown to stabilise the gravitational overturning instability at non-zero streamwise wavenumbers (Gallagher \& Mercer 1965; Kelly 1992; Jerome et al. 2012). The same stabilisation mechanism is also found to act in the present study. In figure 23 , we show a neutral stability curve computed by artificially suppressing the advection transport term of $\left.L_{C}\right|_{\beta=0}$ in (5.7) at $S=8$, and compare it with the original one given in figure $8(\mathrm{c})$. The inhibition of the advection term results in a significant amount of destabilisation at $\alpha<10$, suggesting that the two-dimensional mode tends to be stabilised by the advection, similarly to RayleighBénard convection in a shear flow (Gallagher \& Mercer 1965). However, we note that the Reynolds number in this case is about $R e=3.35$. This value is considerably smaller than 

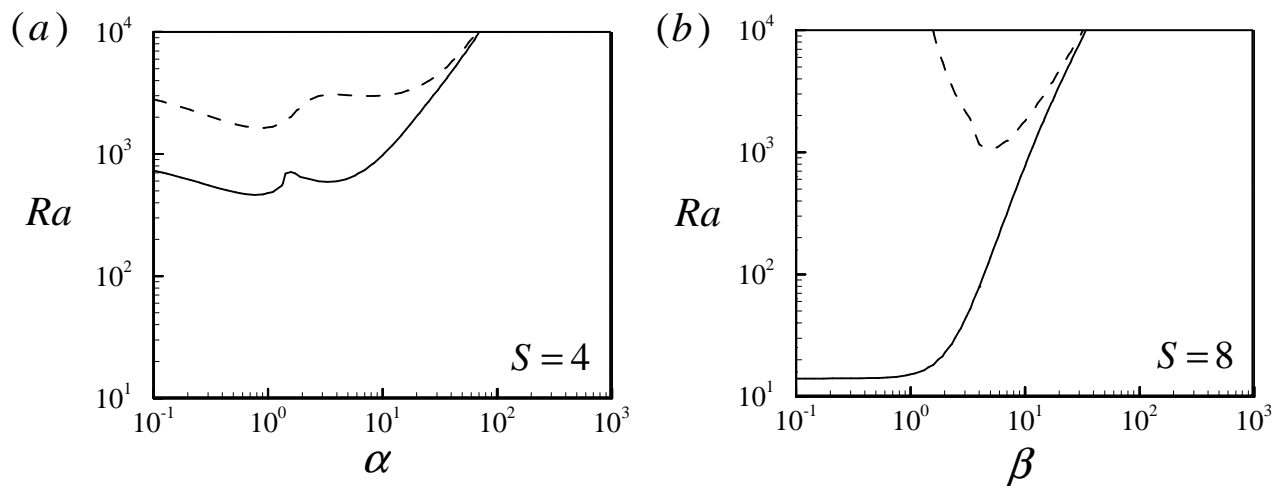

FIGURE 24. Neutral stability curves for $(a)$ the two-dimensional $(\alpha \neq 0$ and $\beta=0)$ and $(b)$ streamwise uniform $(\alpha \neq 0$ and $\beta=0)$ modes with ( -$)$ and without $(----)$ the gravitational term $\left(\tilde{v} d n_{0} / d y\right)$.

$R e \gtrsim O(10)$, at which the stabilising effect begin to appear in Rayleigh-Bénard convection (Gallagher \& Mercer 1965). However, this is presumably due to the large Schmidt number $(\mathrm{Sc}=50)$ in the present system: for example, in Gallagher \& Mercer $(1965)$, the Prandtl number, which is equivalent to the Schmidt number here, is only $\operatorname{Pr}=1$. Finally, we should emphasise that the stabilising effect would be active only at relatively high Reynolds numbers. Therefore, a suspension with a relatively weak shear rate and/or a small depth would not exhibit this effect.

\subsection{Destabilisation by shear - the enhanced gravitational overturning and the reduced overstability}

The introduction of shear has also been found to destabilise the suspension, as shown in figures 8 and 9 . The destabilisation typically appears at $\alpha<\alpha_{b}$, implying that it is presumably associated with the gravitational overturning mechanism. To check this idea, we inhibit the gravitational term $\left(\hat{v} d n_{0} / d y\right)$ and recalculate the neutral stability curves. Figure 24 compares the original neutral stability curves with those in the absence of the gravitational term. When the gravitational term is artificially inhibited, neither the two-dimensional nor the streamwise uniform modes reveal such destabilisation (the dashed lines in figure $24 a$ and $b$ ). This confirms that the destabilisation is indeed due to gravitational overturning in the presence of shear. Furthermore, it suggests that the destabilisation probably originates from the increase of the thickness of the unstably stratified layer with the increase of $S$ (i.e. the decrease of $\kappa$ with $S$ as in figure 7 ). We have shown that the gravitational instability is highly localised near the region where $n_{0}(y)$ is concentrated (e.g. figure 11). Therefore, when the length scale of this locally concentrated layer is increased by the shear, it also increases the effective Rayleigh number of the system, leading to the destabilising effect. From this viewpoint, it is interesting to note that the destabilisation does not occur below $\mathrm{Ra} \sim O(10)$ even with different parameters (see also figures 16-18).

In addition to the destabilisation by the gravitational overturning, there is another independent destabilisation mechanism. This mechanism is found to be associated with the oscillatory mode discussed in $\S 4.4$. It has been thought that the oscillatory instability appears due to overstability caused by the gyrotactic behavior of the cells interacting with the solid boundary. We start by discussing the physical mechanism of the overstability with a fuller explanation than that given by Hill et al. (1989). Figure 25 (a) shows a schematic diagram of the appearance of the oscillatory instability. Imagine that a pair of 
(a)

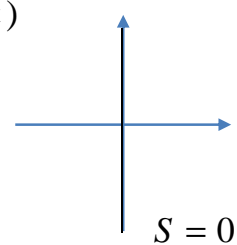

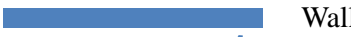

$B_{D} \longleftarrow A$

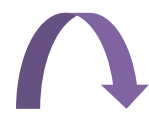

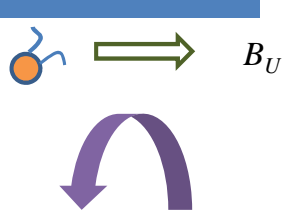

(b)

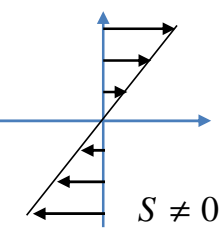

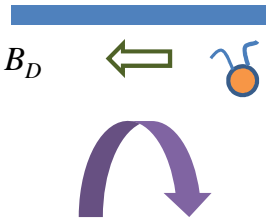

Destabilizing
Wall

A

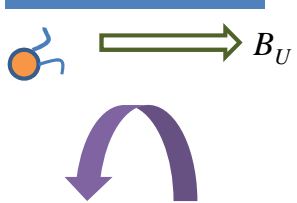

Stabilizing

FiguRE 25. A proposed destabilisation mechanism by the interaction of the oscillatory modes with the shear: $(a) S=0 ;(b) S \neq 0$.

rolls are created by instability in the suspension. The rolls create shear near the upper wall, and the shear would change the swimming direction of the cell at this location due to gyrotaxis. Therefore, the roll with the clockwise rotation would drive the cells to swim from the downwelling $(A)$ to the downstream-upwelling region $\left(B_{D}\right)$, while that with the counter-clockwise rotation would lead the cells to swim from the downwelling $(A)$ to the upstream-upwelling region $\left(B_{U}\right)$. However, the upwelling regions are generally denser than the downwelling region (see e.g. figure 11a). Therefore, this leads to diffusion fluxes against the swimming direction near the upper wall. The oscillatory mode appears when the swimming motions of the cells create fluid motion by overcoming the diffusion fluxes. Therefore, the eigenfunction of a typical oscillatory mode exhibits fluid motions at the top (Hill et al. 1989). This explains why the oscillatory instability often appears at small wavenumbers (see figure 13). Furthermore, the fluid motions brought about by the swimming of the cell can be created either by a clockwise rotating roll or by a counter-clockwise rotating one; one can therefore expect two oscillatory modes, one of which moves upstream and the other propagates downstream. This is consistent with the observation in figure 13. Finally, we note that the oscillatory modes appear due to the gyrotactic nature of the cell. Therefore, it has been observed for parameter values that lead to a relatively large effect of gyrotaxis (Hill et al. 1989; Williams \& Bees 2011). This is also seen in the present study: for example, the decrease of the gyrotaxis control parameters $G_{1}$ and $G_{2}$ leads to a diminishing of the oscillatory mode (figure 17). This also explains why the oscillatory instabilities of the streamwise uniform mode gradually disappear with an increase of the shear rate (figure 15).

Once the oscillatory modes appear, the fluid motion created by the swimming cells should be against the direction of the rolls near the upper wall (figure $25 a$ ). This implies that the appearance of the oscillatory mode would play a stabilising role. It is interesting to note that the growth rate of the oscillatory mode is typically fairly low compared to that of the stationary one: for example, the neutral stability curve with the oscillatory mode in figure $8(a)$ exhibits a much higher critical Rayleigh number than that without the oscillatory mode in figure $17(a)$ at $\alpha \lesssim 10$ (note that the only difference between 
the two cases is in the gyrotaxis control parameters $G_{1}$ and $G_{2}$ ). The same behaviour was also observed in Hill et al. (1989) and Williams \& Bees (2011). Now, we consider the oscillatory modes in the presence of a weak shear rate, as depicted in figure $25(b)$. The imposed shear would tilt the swimming direction of the cell more downstream. Therefore, the number of the cells moving from the region $A$ to $B_{U}$ would increase whereas that from $A$ to $B_{D}$ would decrease, implying that the stabilising role of the upstream-moving mode would be enhanced, while that of the downstream-moving one would be weakened. This explains why the upstream-moving mode is stabilised with an increase of shear while the downstream-moving one is destabilised in figure 13. However, it should be pointed out that this scenario is probably important only at relatively small $S$, as the gyrotactic effect on the instability formation diminishes at high shear rate (figure $22 a$ ).

\subsection{Comparison with Rayleigh-Bénard convection in uniform shear flow}

Bioconvection patterns have often been compared with those in Rayleigh-Bénard convection because of the remarkable similarity between them. Here, we therefore also aim to compare the role of shear in Rayleigh-Bénard convection with that in bioconvection. In Rayleigh-Bénard convection, the presence of shear stabilises only the streamwise varying modes $(\alpha \neq 0)$, leading to the formation of rolls aligned with the shear (Kelly 1992). Similar shear-aligned rolls are also expected in bioconvection (figure 10), but bioconvective instability exhibits much richer dynamical behaviour than Rayleigh-Bénard convection. First, in bioconvection, the structure of the unstable stratification is highly dependent on the shear rate, as the shear can disturb the up-swimming of individual cells (figure 7 ). In particular, we have shown that this feature can lead to destabilisation. However, this does not appear in Rayleigh-Bénard convection, as the source of the unstable stratification is the heat flux from the lower wall. Second, bioconvecton in a suspension of bottom-heavy swimmers is caused not only by the gravitational overturning but also by the gyrotaxis of the given swimmer. Specifically, the latter plays an important role in generating instability at high wavenumbers, and this is also found to contribute to the formation of shear-aligned rolls. Lastly, in bioconvection, very strong shear can completely inhibit the instability. However, in Rayleigh-Bénard convection, the shear is not able to control the instability of the streamwise uniform structure because the linearsed equation for the streamwise uniform mode is completely decoupled from the shear (Kelly 1992).

\subsection{Comparison with experiment}

In spite of many interesting findings here, there has been limited experimental work to investigate the role of shear in bioconvection. To the best of our knowledge, the only experimental work which allows us to make a comparison is by Croze et al. (2010). In this study, the experimental set-up consists of a horizontal pipe with circular cross section, filled with a suspension of $C$. augustae. To introduce a shear in the suspension, the authors applied a flow through the pipe. The shear rate tested is in a relatively narrow range because it was restricted to be smaller than the value leading to tumbling of a deterministic cell (i.e. $1 / B)$.

The flow configuration in the present study differs from that in Croze et al. (2010), since it is designed to understand the simplest case (i.e. uniform shear). For this reason, only qualitative comparison is made. Since the test section of Croze et al. (2010) is circular, it is appropriate to interpret their bioconvection pattern as quasi two-dimensional. Therefore, we only compare the results for the two-dimensional mode $(\alpha \neq 0$ and $\beta=0)$. We have shown that, for a given Rayleigh number (i.e. averaged cell concentration $N$ ), the increase of shear rate suppresses the instability at high wavenumbers. This yields a decrease of the wavenumber for the largest growth rate, implying that the wavelength of 
the most unstable two-dimensional mode increases with the shear rate (figure 10). This is seen to be consistent with the experimental observation, where the average spacing between elements of the pattern is shown to increase with the shear rate. However, it should be pointed out that the wavelength computed in the present study deals only with the initial stage of bioconvection whereas the one in the experiment is measured from the fully developed bioconvection pattern. The structure of the eigenfunctions in the present study shows that the shear tilts the pattern of instability, and this is also observed in the experiment; this is not surprising because it is just a consequence of advection by the shear. Finally, in Croze et al. (2010), the pattern at a low cell concentration showed diminishingly small intensity when the through flow was sufficiently strong. In the present study, we have shown that the shear significantly reduces the growth rate at high wavenumbers while it is destabilising at low wavenumber. We note that the increase in the growth rate by the destabilisation is considerably smaller than the decrease at high wavenumbers. Therefore, the present results suggest that the shear may significantly decrease the intensity of the bioconvection although the convection pattern could persist at shear rates smaller than $O(1 / B)$. This also appears to be consistent with the experiment, but care needs to be taken as the present analysis is limited to small perturbations.

In spite of the encouraging comparison with experimental data, the present results are only qualitatively valid due to some approximations and assumptions discussed previously. For example, the setting of a constant correlation time scale $\tau$ at different shear rates and a constant rotational diffusivity are not obviously realistic. However, the physical processes discussed in the present study have been found to be robust since the use of different parameter values does not yield any qualitative difference. These findings need to be tested by experiments, which can provide quantitative measurement at a wide range of shear rates.

\section{Acknowledgements}

We are grateful to M. A. Bees, O. A. Croze, R. E. Goldstein, and P. J. Schmid for their stimulating discussion on this work. Y.H. is also grateful to S. Furlan, who showed the experimental data on the rotational diffusivity of the gyrotactic cell under uniform vorticity. Y.H. is supported by the European Commission through a Marie Curie fellowship.

\section{Appendix A. Approximation of the Fokker-Planck equation}

Equation (2.7) without any omissions is given as follows:

$$
\begin{aligned}
& \frac{\partial f}{\partial t^{*}}+\left(\mathbf{u}^{*} \cdot \nabla^{*}\right) f+\nabla_{e} \cdot\left[\frac{1}{2 B}[\mathbf{j}-(\mathbf{j} \cdot \mathbf{e}) \mathbf{e}] f+\frac{1}{2} \mathbf{\Omega}^{*} \wedge \mathbf{e} f+\alpha_{0} \mathbf{e} \cdot \mathbf{E}^{*} \cdot\left(\mathbf{I}^{*}-\mathbf{e e}\right)\right]-D_{R}^{*} \nabla_{e}^{2} f \\
& +\left[\left(V_{c}^{*} \mathbf{e}-V_{s}^{*} \mathbf{j}\right) \cdot \nabla^{*}\right] f+\frac{1}{n} \nabla^{*} \cdot\left[V_{c}^{*} n(\mathbf{e}-\langle\mathbf{e}\rangle) f\right]+\nabla^{*} \cdot\left(\mathbf{D}_{T}^{*} \cdot \nabla^{*} f\right)+\frac{2}{n}\left(\mathbf{D}_{T}^{*} \cdot \nabla^{*} n\right) \cdot \nabla^{*} f \\
& =0 .
\end{aligned}
$$

When $V_{c}^{*} / U_{0}^{*} \sim O(\epsilon)$ and $U_{0}^{*} / h \sim O(1 / B)$, the top line becomes $O(1 / B)$. Here, note that the rotational diffusivity term cannot be neglected because $D_{R}^{*} \sim O(1 / B)$ for $\lambda(=$ $2.2) \sim O(1)$. On the other hand, the first and the second terms in the second line are $O(\epsilon / B)$, and the third and the fourth terms are $O\left(\epsilon^{2} / B^{2}\right)$. This implies that the terms describing transport by the swimming velocity of the cell and translational diffusivity will be negligible when $\epsilon \rightarrow 0$. For a given shear rate $U_{0}^{*} / h \sim O(1 / B)$, this condition is 


\begin{tabular}{ccccc} 
& \multicolumn{2}{c}{ for $\Lambda_{0}$} & for $D_{e}$ \\
$\mathrm{Pe}_{c}$ & $\mathrm{Pe}_{0, c r}$ & $S_{c r}$ & $\mathrm{Pe}_{0, c r}$ & $S_{c r}$ \\
1.34 & $\simeq 8$ & $\simeq 8.9$ & $\simeq 6 \quad \simeq 6.7$ \\
6.7 & $\simeq 1000$ & $\simeq 44.5$ & $\simeq 300$ & $\simeq 13.4$
\end{tabular}

TABLE 3 . The critical shear rates $S_{c r}\left(=2 U_{c l}^{*} / D_{R}^{*} h\right)$ above which the present approach begins to deviate from the generalised Taylor dispersion theory based on the computation of the drift of the cell dispersion from the mean velocity $\left(\Lambda_{0}\right)$ and the effective diffusivity $\left(D_{e}\right)$. Here, the values of $\mathrm{Pe}_{c}$ and $\mathrm{Pe}_{0, c r}$ are taken from figure 12 in Croze et al. (2013). We also note that the data for $\mathrm{Pe}_{c}=33.5$ in this figure is omitted due to the lack of the precise values of $\mathrm{Pe}_{0, c r}$.

satisfied if the depth of the suspension becomes

$$
h \gg V_{c}^{*} B=0.021 \mathrm{~cm} .
$$

Therefore, in typical experimental conditions (i.e. $h \simeq 0.5 \sim 1 \mathrm{~cm}$ ), the contribution of the second line of (A 1) would be negligible.

\section{Appendix B. Estimation of the shear rate for comparison with the generalised Taylor dispersion theory}

As mentioned in $\S 2$, in practice, the present approach is identical to that by Pedley \& Kessler (1990) who used (2.6) as an approximation of the diffusivity tensor. Recently, Croze et al. (2013) compared this approach with the generalised Taylor dispersion theory and assessed both of them using dispersion data obtained by simulating a number of random walking (non-interacting) gyrotactic particles $\left(N=2 \times 10^{5}, 10^{6}\right)$ in pressure-driven channel flows. They used the drift of the cell dispersion from the mean velocity $\left(\Lambda_{0}\right)$ and the effective diffusivity $\left(D_{e}\right)$ for the assessment (for further details, see Croze et al. (2013)). They showed that, for a given dimensionless swimming velocity represented by the Péclet number $\mathrm{Pe}_{c}=D_{R}^{*} h / V_{c}^{*}$ (Péclet number), both $\Lambda_{0}$ and $D_{e}$ calculated by the present approach deviate from those from the generalised Taylor dispersion theory when the dimensionless centerline flow velocity of the channel $\mathrm{Pe}_{0}=U_{c l}^{*} D_{R}^{*} h / V_{c}^{* 2}\left(U_{c l}\right.$ is the centerline velocity) exceeds a certain value: i.e. $\mathrm{Pe}_{0}>\mathrm{Pe}_{0, c r}$. Table 3 summarises the critical $\mathrm{Pe}_{0, c r}$, above which $\Lambda_{0}$ and $D_{e}$ computed by the present approach begin to exhibit non-negligible deviation from those from the generalised Taylor dispersion theory. We note that, in the pressure-driven laminar channel flow, the maximum dimensionless shear rate becomes $2 \mathrm{Pe}_{0}$ due to its parabolic velocity profile (they neglected gravity). Conversion of the shear rate $2 \mathrm{Pe}_{0}$ into the dimensionless shear rate $S$ with $D_{R}^{*}$ and $h$ is straightforwardly obtained using the relation $S=2 \mathrm{Pe}_{0} / \mathrm{Pe}_{c}^{2}$. Therefore, the critical dimensionless shear rate $S_{c r}$ above which the present approach deviates from the generalised Taylor dispersion theory is given by

$$
S_{c r}=\frac{2 \mathrm{Pe}_{0, c r}}{\mathrm{Pe}_{c}^{2}}
$$

As seen in table $3, S_{c r} \gtrsim O(10)$, justifying the present approach compared to the generalised Taylor dispersion theory for the shear rate of the interest.

\section{REFERENCES}

Bearon, R. N., Bees, M. A. \& Croze, O. A. 2012 Biased swimming cells do not disperse 
in pipes as tracers: a population model based on microscale behaviour. Phys. Fluids 24, 121902.

Bees, M. A. \& Hill, N. A. 1997 Wavelengths of bioconvection patterns. J. Exp. Biol. 10, $1515-1526$.

BeEs, M. A. \& HiLl, N. A. 1998 Linear bioconvection in a suspension of randomly swimming, gyrotactic micro-organisms. Phys. Fluid 10 (8), 1864-1881.

Bees, M. A., Hill, N. A. \& Pedley, T. J. 1998 Analytical approximations for the orientation distribution of small dipolar particles in steady shear flows. J. Math. Biol. 36, 269-298.

Butler, K. M. \& FARrell, B. F. 1992 Three-dimensional optimal perturbations in viscous shear flow. Phys. Fluids A 4, 1637-1650.

Childress, S., Levandowsky, M. \& Spiegel, E. A. 1975 Pattern formation in a suspension of swimming micro-organisms. J. Fluid Mech. 69, 591-613.

Croze, O. A., Ashraf, E. E. \& Bees, M. A. 2010 Sheared bioconvection in a horizontal tube. Phys. Biol. 7, 046001.

Croze, O. A., Sardina, G., Ahmed, M., Bees, M. A. \& Brandt, L. 2013 Dispersion of swimming algae in laminar and turbulent channel flows: consequences for photobioreactors. J. Royal Soc. Interface 10, 20121041.

Dombrowski, C., Lewellyn, B., Pesci, A. I., Restrepo, J. M., Kessler, J. O. \& GoldSTEIN, R. E. 2005 Coiling, entrainment, and hydrodynamic coupling of decelerated fluid plumes. Phys. Rev. Lett. 95, 184501.

Drazin, P.G. \& ReID, W.H. 1981 Hydrodynamic Stability. Cambridge Univ. Press.

Durham, W. M., Kessler, J. O. \& Stocker, R. 2009 Disruption of vertical motility by shear triggers formation of thin phytoplankton layers. Science 323, 1067-1070.

Ellingsen, T. \& Palm, E. 1975 Stability of linear flow. Phys. Fluids 18, 487.

Furlan, S., Pedley, T. J. \& Goldstein, R. E. 2013 Orientational giant diffusion in gyrotatic microorganisms In preparation.

Gallagher, A. P. \& Mercer, A. McD. 1965 On the behavior of small disturbance in plane couette flow with a temperature gradient. Proc. R. Soc. London. 286, 117-128.

Hill, N. A. \& BeEs, M. A. 2002 Taylor dispersion of gyrotactic swimming micro-organisms in a linear flow. Phys. Fluid 14, 2598-2605.

Hill, N. A. \& HäDER, D.-P. 1997 A biased random walk model for the trajectories of swimming micro-organisms. J. Theor. Biol. 186, 503-526.

Hill, N. A. \& Pedley, T. J. 2005 Bioconvection. Fluid Dyn. Res. 37, 1-20.

Hill, N. A., Pedley, T. J. \& Kessler, J. O. 1989 Growth of bioconvection patterns in a suspension of gyrotactic micro-organisms in a layer of finite depth. J. Fluid Mech. 208, 509-543.

Hwang, Y. \& Cossu, C. 2010 Amplification of coherent streaks in the turbulent Couette flow: an input-output analysis at low Reynolds number. J. Fluid Mech. 643, 333-348.

IsHIKAWA, T. 2012 Vertical dispersion of model microorganisms in horizontal shear flow. J. Fluid Mech. 705, 98-119.

Jeffery, G. B. 1922 The motion of ellipsoidal particles immersed in a viscous fluid. Proc. $R$. Soc. Lond. A 102, 161-179.

Jerome, J. J. S., Chomaz, J.-M. \& Huerre, P. 2012 Transient growth in rayleigh-benardpoiseuille/couette convection. Phys. Fluids 24, 044103.

Kantsler, V., Dunkel, J., Polin, M. \& Goldstein, R. E. 2013 Ciliary contact interactions dominate surface scattering of swimming eukaryotes. Proc. Nat. Acad. Sci. USA 110,11871192.

Kelly, J. E. 1992 The onset and development of thermal convection in fully developed shear flows. Adv. Appl. Mech 31, 35-112.

Kessler, J. O. 1984 Gyrotactic buoyant convection and spontaneous pattern formation in algal cell cultures. In Non-Equilibrium Cooperative Phenomena in Physics and Related Fields (ed. M. G. Verlarde), pp. 241-248. Plenum.

Kessler, J. O. 1985 Hydrodynamics focusing of motile algal cells. Nature 315, 218-220.

Kessler, J. O. 1986 Individual and collective dynamics of swimming cells. J. Fluid Mech. 173, 191-205.

Koch, D. L. \& Shaqfen, E. S. G. 1989 The instability of a dispersion of sedimenting spheroids. J. Fluid Mech. 209, 521-541. 
Malena, A. \& Frankel, I. 2003 Generalized taylor dispersion in suspensions of gyrotactic swimming micro-organisms. J. Fluid Mech. 490, 99-127.

Pedley, T. J. 2010 a Collective behaviour of swimming micro-organisms. Exp. Mech. 50, 12931301.

Pedley, T. J. $2010 b$ Instability of uniform microorganism suspensions revisited. J. Fluid Mech. 647, 335-359.

Pedley, T. J., Hill, N. A. \& Kessler, J. O. 1988 The growth of bioconvection patterns in a uniform suspension of gyrotactic micro-organisms. J. Fluid Mech. 195, 223-237.

Pedley, T. J. \& Kessler, J. O. 1987 The orientation of spheroidal microorganisms swimming in a flow field. Proc. R. Soc. Lond. B 231, 47-70.

Pedley, T. J. \& Kessler, J. O. 1990 A new continuum model for suspensions of gyrotactic micro-organisms. J. Fluid Mech. 212, 155-182.

Pedley, T. J. \& Kessler, J. O. 1992 Hydrodynamic phenomena in suspensions of swimming micro-organisms. Annu. Rev. Fluid Mech. 24, 313-358.

Reddy, S. C. \& Henningson, D. S. 1993 Energy growth in viscous channel flows. J. Fluid Mech. 252, 209-238.

Saintillan, D., Shaqfeh, E. S. G. \& Darve, E. 2006 The growth of concentration fluctuations in dilute dispersions of orientable and deformable particles under sedimentation. J. Fluid Mech. 553, 347-388.

Saintillan, D. \& Shelley, M. J. 2007 Orientiational order and instabilities in suspensions of self-locomoting rods. Phys. Rev. Lett. 99, 058102.

Saintillan, D. \& Shelley, M. J. 2008 Instabilities and pattern formation in active particle suspensions: kinetic theory and continuum simulations. Phys. Rev. Lett. 100, 178103.

Schmid, P.J. 2007 Nonmodal stability theory. Annu. Rev. Fluid Mech. 39, 129-162.

Schmid, P. J. \& Henningson, D. S. 2001 Stability and Transition in Shear Flows. New York: Springer.

Simha, R. A. \& Ramaswamy, S. 2002 Hydrodynamic fluctuations and instabilities in ordered suspensions of self-propelled particles. Phys. Rev. Lett. 89, 058101.

Vladimirov, V. A., Denissenko, P. V., Pedley, T. J., We, M. \& Mosklaev, I. S. 2000 Algal motility measured by a laser based tracking method. Mar. Freshwater Res. 51, 589600 .

Vladimirov, V. A., Denissenko, P. V., Pedley, T. J., We, M. \& Zakhidova, I. S. 2004 Measurement of cell velocity distributions in populations of motile algae. J. Exp. Biol 207, $1203-1216$.

Weideman, J. A. C. \& Reddy, S. C. 2000 A MATlAB Differentiation Matrix Suite. ACM Trans. Math. Soft. 26, 465-519.

Williams, C. R. \& Bees, M. A. 2011 Photo-gyrotactic bioconvection. J. Fluid Mech. 678, $41-86$. 